\title{
Parametric controllability of the personalized PageRank: Classic model vs biplex approach
}

Cite as: Chaos 30, 023115 (2020); https://doi.org/10.1063/1.5128567

Submitted: 21 September 2019 . Accepted: 20 January 2020 . Published Online: 04 February 2020

Julio Flores (D), Esther García (D), Francisco Pedroche (D), and Miguel Romance (iD)
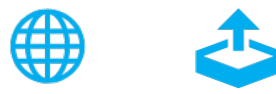

\section{Sollight Highlights of the best new research} in the physical sciences 


\title{
Parametric controllability of the personalized PageRank: Classic model vs biplex approach
}

\author{
Cite as: Chaos 30, 023115 (2020); doi: 10.1063/1.5128567 \\ Submitted: 21 September 2019 . Accepted: 20 January 2020 . \\ Published Online: 4 February 2020

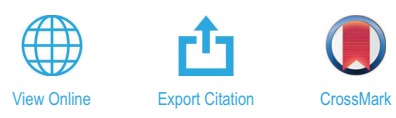

Julio Flores, ${ }^{1,2}$ (D) Esther García, ' (D) Francisco Pedroche, ${ }^{3}$ (D) and Miguel Romance ${ }^{1,2,4}$ (D)

\author{
AFFILIATIONS \\ ${ }^{1}$ Department of Applied Mathematics, Rey Juan Carlos University, 28933 Madrid, Spain \\ ${ }^{2}$ Center for Computational Simulation, Technical University of Madrid, 28223 Pozuelo de Alarcón, Madrid, Spain \\ ${ }^{3}$ Institut de Matemàtica Multidisciplinària, Universitat Politècnica de València, València, Spain \\ ${ }^{4}$ Data, Networks and Cybersecurity Research Institute, 28028 Madrid, Spain
}

\begin{abstract}
Measures of centrality in networks defined by means of matrix algebra, like PageRank-type centralities, have been used for over 70 years. Recently, new extensions of PageRank have been formulated and may include a personalization (or teleportation) vector. It is accepted that one of the key issues for any centrality measure formulation is to what extent someone can control its variability. In this paper, we compare the limits of variability of two centrality measures for complex networks that we call classic PageRank (PR) and biplex approach PageRank (BPR). Both centrality measures depend on the so-called damping parameter $\alpha$ that controls the quantity of teleportation. Our first result is that the intersection of the intervals of variation of both centrality measures is always a nonempty set. Our second result is that when $\alpha$ is lower that 0.48 (and, therefore, the ranking is highly affected by teleportation effects) then the upper limits of PR are more controllable than the upper limits of BPR; on the contrary, when $\alpha$ is greater than 0.5 (and we recall that the usual PageRank algorithm uses the value 0.85), then the upper limits of PR are less controllable than the upper limits of BPR, provided certain mild assumptions on the local structure of the graph. Regarding the lower limits of variability, we give a result for small values of $\alpha$. We illustrate the results with some analytical networks and also with a real Facebook network.
\end{abstract}

Published under license by AIP Publishing. https://doi.org/10.1063/1.5128567

It is known that PageRank-type centralities were used long before Google became a famous searcher, but it is also recognized that the reputation of this company promoted the study and use of classic tools like Markov chains and Perron Frobenius theorems. Currently, new PageRank-type centralities are used extensively. In particular, we focus on the biplex approach PageRank centrality, ${ }^{52}$ and we study the limits of variability of this centrality when compared to the classic PageRank centrality. ${ }^{49}$ Our main conclusion is that when the teleportation effect is not too big, then the upper limits of the biplex approach PageRank are lower than those of the classic PageRank. That is, if one wants to bias the ranking to any particular node by using the personalization vector-that is, one of the main ingredients of any PageRank centrality-then this task is more easily done by using the classic PageRank, and, therefore, we conclude that in this case, the classic PageRank centrality is more controllable.

\section{INTRODUCTION}

The fundamental tool in this paper is matrix power series. We first make a small historical review of the use of matrix power series in connection with the concept of centrality measures (measures introduced to account for the important people on social networks).

The pioneering works of Festinger ${ }^{22}$ and $\mathrm{Katz}^{38}$ on the introduction of matrix analysis to study centrality-related problems in social networks still maintain the validity of their approaches. In Ref. 22, the author points out that when the number of members is very high, the visual depiction of the graph is not enough to give ideas about the interconnections of the nodes. To overcome this problem, the author makes use of matrices and shows that the powering of the adjacency matrix $A$ and its sums (that is, $\sum_{i=1}^{k} A^{i}$ ) give us an idea of how many people will receive a piece of information in $k$ or less steps. It is also worth noting the relation that the author makes between reorderings of the adjacency matrix and the detection of communities (in this respect, one immediately thinks of the works of Fiedler published years later; see, e.g., Ref. 23). Regarding the works of Katz, in Ref. 38, it is considered that the importance of a node is not only a matter of the quantity of votes (links) that it receives but also the importance of the people who are voting for that node. He used matrix algebra to make a model. Nowadays, it is considered (see Ref. 47) that the PageRank model is a modification of Katz centrality, and this fact has given new insight into the historical 
works of Katz. In this small account of pioneering works on centrality measures, it is also mandatory to cite the seminal work of Babelas because, although matrices are not used explicitly, it shows a systematic approach to define concepts such as the shortest distance between nodes (which are called cells) and, surprisingly, the introduction of open cells that are strongly reminiscent of the concept of dangling node introduced 50 years later by the founders of Google. ${ }^{4}$ It is also worthy to recall that the first algorithms to find shortest paths are posterior in time, since they can be traced back to 1956 (see Refs. 24 and 39). It is also important to remark that in current times, some authors use not the adjacency matrix itself but functions of the adjacency matrix to construct power series in order to solve problems about centrality_or more particularly_communicability (see, e.g., Ref. 21).

As we have seen so far, the original works put two problems laid down on the table. On the one hand, they established that matrix algebra was the key tool to treat these centrality questions. In more detail, they used matrix power series. On the other hand, they put some questions that remain open and involve more quantity and type of data. In fact, analysis of complex networks has a central role in the contemporary global economy. In particular, we can think of big corporations that use enormous social networks and want to know who are the important users, how information is transmitted and how to avoid issues such as falls down, infrastructure attacks, ${ }^{60}$ or fake news. ${ }^{3}$

From this initial centrality measures, there have been a lot of studies trying to define precisely the nature and function of any centrality measure together with the way in which they can be computed (see, e.g., Refs. 11, 13, 14, 26, and 53). In this paper, we are interested in PageRank centrality, which can be formulated as a linear system or as a power matrix series, and it can be computed efficiently as a Markov chain. ${ }^{12,27,40,45}$

Studies about the correlation of PageRank with in-degree were conducted several years ago (see Ref. 25), but they do not stop research from using PageRank in different fields. We are interested in the application of the PageRank algorithm by using different personalization vectors, what is commonly called personalized PageRank. ${ }^{30}$ This personalization gives the PageRank algorithm more flexibility, and it can result in more differences in the ranking with respect to the in-degree approximation. Moreover, PageRank centrality is a currently active field as it is shown by the quantity of new papers on the topic and by the variety of themes. For example, PageRank has been applied to analyze the human brain modeled as a directed graph of neuron links. ${ }^{57}$ PageRank is most appreciated when using incomplete data as generally happens in biological networks as protein interaction networks. ${ }^{36,37}$ PageRank is also used as a reference model to compare rankings that use new techniques. ${ }^{18}$ PageRank has also recently been used to analyze opinion formation, ${ }^{28}$ for networks that change with time while preserving their nodes, ${ }^{42}$ to analyze risk in financial networks, ${ }^{62}$ to include the concept of trust on weighted social networks, ${ }^{15}$ to study the vulnerability of gas and electricity networks, ${ }^{55}$ to rank citation networks, ${ }^{44}$ and to rank products following multiple criteria in dynamic markets. ${ }^{54}$ There are also new numerical methods for the PageRank algorithm (see Ref. 59 for the use of regular splittings, ${ }^{31}$ for a use of GMRES (generalized minimal residual method), ${ }^{56}$ for low-rank factorizations, and ${ }^{46}$ for parallelization).
New extensions to PageRank have also been invented to account for the new paradigm of multiplex networks, see Refs. 9, $10,16,32,34,35$, and 52 . Of course, other centrality measures have been adapted to multiplex networks too (see, e.g., HITS, ${ }^{5}$ eigenvector centrality, ${ }^{8,58}$ and other measures ${ }^{17}$ ).

In this paper, we focus on comparing the intervals of variation of two centrality measures. On the one hand, the variation of the classic PageRank (PR) was studied in Ref. 29, and on the other hand, the variation of the biplex approach PageRank (as defined in Ref. 52) was studied in Ref. 50. The biplex approach PageRank (BPR) was defined with the aim of establishing a building block to construct a definition of Multiplex PageRank (see Ref. 52 for details) but, as a centrality measure itself, BPR has shown some interesting properties. BPR reduces to classic PageRank for some limit situations, it also correlates well with classic PageRank for the tested networks so far, and it has been used in connection to other centrality measures like the works in Refs. 1 and 2. Moreover, BPR can be used as an analogy to construct other centrality measures like in Ref. 51.

The main interest of this paper is twofold. First, it gives an idea of how well the BPR is a good approach to PR (and, as a by-product, it is also evidence of its use as building block to construct a multiplex PageRank as in Ref. 52). Second, the fact of knowing the variation of a centrality measure can be translated to the degree of controllability of that measure (and controlling centralities is an active line of research, as it is shown in Refs. 4, 41, and 48).

The rest of the paper has the following structure. In Sec. II, we give the basic definitions to introduce the limits of variation of $P R$ and BPR and prove some technical lemmas that will be useful later. In this section, we also show that the intervals for PR and BPR always intersect. In Sec. III, we study the relative position of the upper limits of the intervals and give a sharp result. For the lower limits of the intervals, we give a result only for small values of the damping factor. In Sec. IV, we give some examples to illustrate the results. The first is a star-shaped network of any number of nodes. The second consists of some synthetic random families of complex networks, and the third is a real Facebook network of 167 nodes. Finally, some conclusions of the work are given in Sec. V.

\section{PRELIMINARIES AND BASIC RESULTS}

Throughout this paper, $P$ will denote the row-stochastic matrix of a complex network with $n$ nodes, no loops, and no dangling nodes.

The Google matrix with personalization vector $\mathbf{v}\left(\mathbf{v} \in \mathbb{R}^{n}\right.$ such that $\mathbf{v}>0$ and $\mathbf{v}^{T} \mathbf{e}=1$, where $\mathbf{e}$ is the all ones vector in $\left.\mathbb{R}^{n}\right)$ and damping factor $\alpha \in(0,1)$ is $G=\alpha P+(1-\alpha) \mathbf{e v}^{T}$. Recall that the (personalized) classic PageRank vector $\pi$ is the unique eigenvector of $G^{T}$ associated with the eigenvalue 1 such that $\pi^{T} \mathbf{e}=1, \pi>0$, and $\pi^{T} G=\pi^{T}$, while the (personalized) classic PageRank of node $i$ is the $i$ th-component of $\pi$. We denote by $\mathcal{P} \mathcal{R}_{\alpha}(i)$ the set of all possible values of the (personalized) classic PageRank of node $i \in\{1, \ldots, n\}$ and fixed $\alpha \in(0,1)$. It is shown in Ref. 29 (Theorem 3.2) that

$$
\mathcal{P} \mathcal{R}_{\alpha}(i)=\left(\min _{j} x_{j i}, x_{i i}\right),
$$

where $X=\left(x_{i j}\right)$ is the matrix given by

$$
X=(1-\alpha)(I-\alpha P)^{-1} .
$$


Recall that matrix $X$ is strictly diagonally dominant of its column entries and the maximum of each column $i$ is achieved in $x_{i i}$, see Ref. 29 (Lemma 2.3).

Similarly (see Ref. 52), if we denote by $\mathcal{P} \mathcal{R} \mathcal{B}_{\alpha}(i)$ the set of all possible values of the (personalized) Biplex approach PageRank of node $i \in\{1, \ldots, n\}$ and fixed $\alpha \in(0,1)$, it is shown in Ref. 50 (Theorem 3.9) that

$$
\mathcal{P} \mathcal{R} \mathcal{B}_{\alpha}(i)=\left(\min _{j} c_{j i}, c_{i i}\right),
$$

where $C=\left(c_{i j}\right)$ is the matrix given by

$$
C=\frac{(1-\alpha)^{2}}{\beta}\left(I-\frac{\alpha}{\beta} P\right)^{-1}((1+\alpha) I-\alpha P),
$$

with $\beta=1-\alpha(1-\alpha)$. Recall that matrix $C$ is also strictly diagonally dominant of its column entries and the maximum of each column $i$ is achieved in $c_{i i}$, see Ref. 29 (Lemma 2.3).

We would like to investigate the relation between these two intervals and decide when one type of PageRank should be preferred over the other.

For all admissible $\mu \in \mathbb{R}$, the operator $(I-\mu P)^{-1}$ will be denoted as $R(\mu)$. In particular, if $0 \leq \mu<1$, we have

$$
R(\mu)=\sum_{k=0}^{\infty} \mu^{k} P^{k} .
$$

Notice that

$$
\begin{gathered}
(I-\alpha P)^{-1}=R(\alpha), \\
\frac{1}{\beta}\left(I-\frac{\alpha}{\beta} P\right)^{-1}=\frac{1}{\beta} R\left(\frac{\alpha}{\beta}\right),
\end{gathered}
$$

so if we use these expressions in (1) and (2), we get that

$$
\begin{gathered}
X=(1-\alpha) R(\alpha), \\
C=(1-\alpha)^{2}\left(I+\frac{\alpha(2-\alpha)}{\beta} R\left(\frac{\alpha}{\beta}\right)\right),
\end{gathered}
$$

and thus, we can express $C-X$ as

$$
(1-\alpha)^{2}\left(I+\frac{\gamma}{\beta(1-\alpha)} R\left(\frac{\alpha}{\beta}\right)\right)-(1-\alpha) R(\alpha) .
$$

Since matrices $X$ and $C$ are expressed in terms of the operator $R(\cdot)$, several properties of such operator are needed and summarized in the following results.

Lemma 2.1. Let $n \in \mathbb{N}, P=\left(p_{i j}\right)$ be the row-stochastic matrix associated with a complex network of $n$ nodes with no loops and no dangling nodes, and let $M_{i}=\max _{j} p_{j i}$ be the maximum of the entries of the ith-column of $P$. Then, for all $i, j \in\{1, \ldots, n\}$ and every $\lambda \in$ $(0,1)$, we have

$$
\begin{aligned}
& \cdot 0 \leq\left(R(\lambda)^{2}\right)_{i j} \leq M_{j} \frac{\lambda(2-\lambda)}{(1-\lambda)^{2}}, \text { if } i \neq j, \\
& \text { - } 1 \leq R(\lambda)_{i i} \leq 1+\frac{M_{i} \lambda^{2}}{1-\lambda}, \text { if } i=j .
\end{aligned}
$$

Proof. (i) Note that

$$
\begin{aligned}
R(\lambda)^{2} & =\left(\sum_{k=0}^{\infty} \lambda^{k} P^{k}\right)^{2}=\sum_{k=0}^{\infty}\left(\sum_{\ell=0}^{k} \lambda^{\ell} P^{\ell} \lambda^{k-\ell} P^{k-\ell}\right) \\
& =\sum_{k=0}^{\infty}(k+1) \lambda^{k} P^{k},
\end{aligned}
$$

so if $i \neq j$, then by using that $P$ is row stochastic, we get that

$$
0 \leq M_{j} \sum_{k=0}^{\infty}(k+1) \lambda^{k}=M_{j} \frac{\lambda(2-\lambda)}{(1-\lambda)^{2}} .
$$

(ii) Since $P$ is a row-stochastic matrix, for every $k \geq 2$, we have that $\left(P^{k}\right)_{i i} \leq M_{i}$, so

$$
\begin{aligned}
1 \leq R(\lambda)_{i i} & =1+\sum_{k=2} \lambda^{k}\left(P^{k}\right)_{i i} \\
& \leq 1+M_{i} \sum_{k=2} \lambda^{k}=1+\frac{M_{i} \lambda^{2}}{1-\lambda} .
\end{aligned}
$$

The following identity is a direct consequence of the First Resolvent Identity [see Ref. 19, p. 566)] and will be used later:

Lemma 2.2. For every $\lambda, \mu$ in the domain of $R(\cdot)$

$$
\mu R(\mu)-\lambda R(\lambda)=(\mu-\lambda) R(\lambda) R(\mu) .
$$

In particular, we will use that

$$
R(\alpha)=\frac{1}{\beta} R\left(\frac{\alpha}{\beta}\right)-\frac{\alpha(1-\alpha)}{\beta} R(\alpha) R\left(\frac{\alpha}{\beta}\right) .
$$

If we denote $\gamma=\alpha(1-\alpha)(2-\alpha)$, by using (5), we get that

$$
\begin{aligned}
\frac{C-X}{1-\alpha} & =(1-\alpha)\left[I+\frac{\alpha(2-\alpha)}{\beta} R\left(\frac{\alpha}{\beta}\right)\right]-R(\alpha) \\
& =(1-\alpha) I+\left[(\gamma-1) I+\frac{\gamma \alpha(1-\alpha)}{\beta} R\left(\frac{\alpha}{\beta}\right)\right] R(\alpha) \\
& =(1-\alpha) I-(1-\gamma)\left[I-\frac{\gamma \alpha(1-\alpha)}{\beta(1-\gamma)} R\left(\frac{\alpha}{\beta}\right)\right] R(\alpha)
\end{aligned}
$$

and thus,

$$
\frac{C-X}{(1-\alpha)^{2}}=I-\frac{1-\gamma}{1-\alpha}\left[I-\frac{\gamma \alpha(1-\alpha)}{\beta(1-\gamma)} R\left(\frac{\alpha}{\beta}\right)\right] R(\alpha) .
$$

We remark that (8) strongly suggests considering the Resolvent Identity (7) for $\alpha$ and $\frac{\alpha}{\beta}$. To this end, some estimations involving $R(\alpha)$ and $R\left(\frac{\alpha}{\beta}\right)$ will be needed.

Lemma 2.3. Given any $n \in \mathbb{N}$, for all $i, j \in\{1, \ldots, n\}$ and every $\alpha \in(0,1)$, we have

$$
\frac{\beta}{1-\alpha} \min _{i} R(\alpha)_{i j} \leq R\left(\frac{\alpha}{\beta}\right)_{i j} \leq \frac{\beta}{1-\alpha} R(\alpha)_{j j} .
$$

Furthermore, if $i \neq j$, then

$$
R(\alpha)_{i j} \leq \alpha R(\alpha)_{j j}
$$


Proof. By Lemma 2.2, we have that

$$
R(\alpha)=\frac{1}{\beta} R\left(\frac{\alpha}{\beta}\right)(I-\alpha(1-\alpha) R(\alpha)),
$$

SO

$$
\begin{aligned}
R\left(\frac{\alpha}{\beta}\right) & =\beta R(\alpha)[I-\alpha(1-\alpha) R(\alpha)]^{-1} \\
& =\beta R(\alpha) \sum_{k=0}^{\infty} \alpha^{k}(1-\alpha)^{k} R(\alpha)^{k} \\
& =\frac{\beta}{1-\alpha} R(\alpha)\left[(1-\alpha) \sum_{k=0}^{\infty} \alpha^{k}[(1-\alpha) R(\alpha)]^{k}\right] \\
& =\frac{\beta}{1-\alpha} R(\alpha) T=\frac{\beta}{1-\alpha} T R(\alpha),
\end{aligned}
$$

for

$$
T=(1-\alpha) \sum_{k=0}^{\infty} \alpha^{k}[(1-\alpha) R(\alpha)]^{k} .
$$

Notice that $T$ is row stochastic because $[(1-\alpha) R(\alpha)]^{k}$ is row stochastic. Thus, on the one hand,

$$
\frac{\beta}{1-\alpha} \min _{i} R(\alpha)_{i j} \leq R\left(\frac{\alpha}{\beta}\right)_{i j}
$$

and on the other hand,

$$
R\left(\frac{\alpha}{\beta}\right)_{i j} \leq \frac{\beta}{1-\alpha} \max _{i} R(\alpha)_{i j}=\frac{\beta}{1-\alpha} R(\alpha)_{j j} .
$$

Now, we denote $\left(y_{i j}\right)=Y=I-\alpha P$ and consider for every $i=1, \ldots, n$

$$
r_{i}(Y)=\sum_{j \neq i} \frac{\left|y_{i j}\right|}{\left|y_{i i}\right|}=\sum_{j \neq i}\left|y_{i j}\right|\left(\text { recall that } y_{i i}=1\right) .
$$

In addition, since $P$ is row stochastic

$$
r_{i}(Y)=\alpha \sum_{j \neq i} p_{i j}=\alpha .
$$

Notice also that $Y$ is strictly row diagonally dominant. Reference 33 (Ex. 2.5.17) follows

$$
\frac{R(\alpha)_{i j}}{r_{i}(Y)} \leq R(\alpha)_{j j}
$$

for all $j=1, \ldots, n$ and $i \neq j$, i.e.,

$$
R(\alpha)_{i j} \leq r_{i}(Y) R(\alpha)_{j j}=\alpha R(\alpha)_{j j} .
$$

Corollary 2.4. Given any $n \in \mathbb{N}$, for all $i, j \in\{1, \ldots, n\}$ and every $\alpha \in(0,1)$, we have

- $\min _{i} R(\alpha)_{i j}=\min _{i \neq j} R(\alpha)_{i j}$ and $\min _{i} x_{i j}=\min _{i \neq j} x_{i j}$

- $\min _{i} R\left(\frac{\alpha}{\beta}\right)_{i j}=\min _{i \neq j} R\left(\frac{\alpha}{\beta}\right)_{i j}$ and $\min _{i} c_{i j}=\min _{i \neq j} c_{i j}$.
Proof. First, notice that if we compute each element of $X$ and $C$ in (3) and (4), then for every $i, j \in\{1, \ldots, n\}$,

$$
\begin{aligned}
& \left\{\begin{array}{l}
x_{i j}=(1-\alpha) R(\alpha) i j, \quad i \neq j, \\
x_{j j}=(1-\alpha) R(\alpha)_{j j} .
\end{array}\right. \\
& \left\{\begin{array}{l}
c_{i j}=\frac{(1-\alpha) \gamma}{\beta} R\left(\frac{\alpha}{\beta}\right)_{i j}, \quad i \neq j \\
c_{j j}=(1-\alpha)^{2}+\frac{(1-\alpha) \gamma}{\beta} R\left(\frac{\alpha}{\beta}\right)_{j j},
\end{array}\right.
\end{aligned}
$$

In order to prove (i), we will proceed by Reductio ad Absurdum. If we assume on the contrary that $\min _{i} x_{i j}=x_{j j}$, which is clearly equivalent to the fact that $R(\alpha)_{i j} \geq R(\alpha)_{i j}$, for all $i \neq j$, then consequently, by Lemma 2.3, we get that

$$
1 \leq \frac{\min _{i \neq j} R(\alpha)_{i j}}{R(\alpha)_{j j}} \leq \alpha<1,
$$

which is a contradiction.

Note that the first part of (ii) is a direct consequence of (i). Indeed, it suffices to notice that $0<\frac{\alpha}{\beta}<1$, for all $\alpha \in(0,1)$. In order to prove the second part of (ii), we will proceed again by Reductio ad Absurdum. If we suppose that $\min _{i} c_{i j}=c_{j j}$, then

$$
1 \leq \frac{\frac{(1-\alpha) \gamma}{\beta} R\left(\frac{\alpha}{\beta}\right)_{i j}}{(1-\alpha)^{2}+\frac{(1-\alpha) \gamma}{\beta} R\left(\frac{\alpha}{\beta}\right)_{j j}} \leq \frac{\frac{(1-\alpha) \gamma}{\beta} R\left(\frac{\alpha}{\beta}\right)_{i j}}{\frac{(1-\alpha) \gamma}{\beta} R\left(\frac{\alpha}{\beta}\right)_{j j}} \leq \frac{\alpha}{\beta}<1,
$$

for all $i \neq j$, again by using Lemma 2.3, which is a contradiction.

Lemma 2.5. Given any $n \in \mathbb{N}$, for all $i, j \in\{1, \ldots, n\}$ and every $\alpha \in(0,1)$, we have

- The following inequalities hold:

$$
\begin{aligned}
\frac{\beta}{(1-\alpha)^{2}} \min _{j} R(\alpha)_{j i} & \leq\left(R\left(\frac{\alpha}{\beta}\right) R(\alpha)\right)_{i i}, \\
\left(R\left(\frac{\alpha}{\beta}\right) R(\alpha)\right)_{i i} & \leq \frac{\beta}{(1-\alpha)^{2}} R(\alpha)_{i i}, \\
\frac{\beta}{(1-\alpha)^{2}} R(\alpha)_{i i} & \leq \frac{\beta}{(1-\alpha)^{2}}\left(R(\alpha)_{i i}\right)^{2} .
\end{aligned}
$$

- $\left(R(\alpha)_{i i}\right)^{2} \leq\left(R\left(\frac{\alpha}{\beta}\right) R(\alpha)\right)_{i i}$.

- The following inequality holds:

$$
\begin{gathered}
R(\alpha)_{i i}-\frac{\gamma \alpha(1-\alpha)}{(1-\gamma) \beta}\left(R\left(\frac{\alpha}{\beta}\right) R(\alpha)\right)_{i i} \\
\quad \leq R(\alpha)_{i i}\left[1-\frac{\gamma \alpha(1-\alpha)}{(1-\gamma) \beta} R(\alpha)_{i i}\right] .
\end{gathered}
$$

Proof. (i) Notice that matrix $W=\frac{(1-\alpha)^{2}}{\beta} R\left(\frac{\alpha}{\beta}\right)$ is row stochastic and since $\max _{j} R(\alpha)_{j i}=R(\alpha)_{i i}$, it follows that

$$
\begin{aligned}
\min _{j} R(\alpha)_{j i} & =e_{i}^{T}\left(\min _{j} R(\alpha)_{j i} \mathbf{1}\right) \leq(W R(\alpha))_{i i} \\
& =e_{i}^{T}\left(W R(\alpha) e_{i}\right) \leq e_{i}^{T}\left(R(\alpha)_{i i} \mathbf{1}\right)=R(\alpha)_{i i},
\end{aligned}
$$

which proves the first two inequalities. The last inequality in (i) is a direct consequence of the fact that $1 \leq R(\alpha)_{i i}$ and hence $R(\alpha)_{i i} \leq$ $(R(\alpha))_{i i}^{2}$, for all $\alpha \in(0,1)$. 
(ii) Note that

$$
\left(R\left(\frac{\alpha}{\beta}\right) R(\alpha)\right)_{i i} \geq R\left(\frac{\alpha}{\beta}\right)_{i i} R(\alpha)_{i i} \geq\left(R(\alpha)_{i i}\right)^{2},
$$

where the condition $i=j$ is essential here.

Finally, (iii) easily follows from (ii).

To conclude this section, we prove a relevant result showing that for every $\alpha \in(0,1)$ and every arbitrary node $i \in\{1, \ldots, n\}$, there is always some overlapping between the intervals $\mathcal{P} \mathcal{R}_{\alpha}(i)$ and $\mathcal{P} \mathcal{R} \mathcal{B}_{\alpha}(i)$.

Theorem 2.6. Let us consider a complex network with $n \in \mathbb{N}$ nodes, no loops and no dangling nodes, let $i \in\{1, \ldots, n\}$ be a node of the complex network. Let us denote by $\mathcal{P} \mathcal{R}_{\alpha}(i)$ and $\mathcal{P} \mathcal{R B}_{\alpha}(i)$ the intervals of all possible values of the Classical and Biplex PageRank respectively of node i for some fixed $\alpha \in(0,1)$. Then, $\mathcal{P} \mathcal{R}_{\alpha}(i) \cap$ $\mathcal{P} \mathcal{R} \mathcal{B}_{\alpha}(i) \neq \emptyset$.

Proof. We will proceed by Reductio ad Absurdum. Given a node $i \in\{1, \ldots, n\}$ and $\alpha \in(0,1)$, we assume that $\mathcal{P} \mathcal{R}_{\alpha}(i) \cap$ $\mathcal{P} \mathcal{R} \mathcal{B}_{\alpha}(i)=\emptyset$. Since the upper bound of the interval $\mathcal{P} \mathcal{R}_{\alpha}(i)$ is $x_{i i}$ and the upper bound of $\mathcal{P} \mathcal{R} \mathcal{B}(i)$ is $c_{i i}$, then either $\min _{j} c_{j i}>x_{i i}$ or $c_{i i}<\min _{j} x_{j i}$. Now, we check both cases:

- Suppose that $\min _{j} c_{j i}>x_{i i}$. From (5), we have that

$$
\frac{\gamma}{\beta} R\left(\frac{\alpha}{\beta}\right)_{j i}-R(\alpha)_{i i}>0 \text { for every } \neq \neq i .
$$

Then, by Lemma 2.3,

$$
\frac{\gamma}{\beta} R\left(\frac{\alpha}{\beta}\right)_{i i} \geq \frac{\gamma}{\beta} R\left(\frac{\alpha}{\beta}\right)_{j i}>R(\alpha)_{i i} \geq \frac{1-\alpha}{\beta} R\left(\frac{\alpha}{\beta}\right)_{i i} .
$$

Notice that $R\left(\frac{\alpha}{\beta}\right)_{i i} \neq 0$ since otherwise $R\left(\frac{\alpha}{\beta}\right)_{i i}=R\left(\frac{\alpha}{\beta}\right)_{j i}=0$ $=R(\alpha)_{i i}$ for all $j$, so $0=\min _{j} c_{j i} \ngtr x_{i i}$. Thus,

$$
\frac{\gamma}{\beta}>\frac{1-\alpha}{\beta}
$$

which makes that $\gamma-(1-\alpha)>0$, but this is a contradiction since for every $\alpha \in(0,1)$

$$
\gamma-(1-\alpha)=(\alpha-1)^{3}<0 .
$$

- Suppose that $c_{i i}<\min _{j} x_{j i}$. From (5), we have that

$$
\begin{aligned}
& (1-\alpha)+\frac{\gamma}{\beta} R\left(\frac{\alpha}{\beta}\right)_{i i}<\min _{j} R(\alpha)_{j i} \\
& \Longleftrightarrow(1-\alpha)+\frac{\gamma}{\beta} R\left(\frac{\alpha}{\beta}\right)_{i i}<\frac{1-\alpha}{\beta} \frac{\beta}{1-\alpha} \min _{j} R(\alpha)_{j i},
\end{aligned}
$$

which implies by Lemma 2.3 that for every $j$,

$$
(1-\alpha)+\frac{\gamma}{\beta} R\left(\frac{\alpha}{\beta}\right)_{i i}<\frac{1-\alpha}{\beta} R\left(\frac{\alpha}{\beta}\right)_{j i} .
$$

Notice that $R\left(\frac{\alpha}{\beta}\right)_{i i} \neq 0$ since, otherwise, $R\left(\frac{\alpha}{\beta}\right)_{j i}=0$ for every $j$, and this would imply that $1-\alpha<0$. Then, for every $1 \leq j \leq n$,

$$
\frac{1-\alpha}{R\left(\frac{\alpha}{\beta}\right)_{i i}}+\frac{\gamma}{\beta}<\frac{1-\alpha}{\beta} \frac{R\left(\frac{\alpha}{\beta}\right)_{j i}}{R\left(\frac{\alpha}{\beta}\right)_{i i}} .
$$

Therefore, by Lemma 2.3,

$$
\frac{1-\alpha}{R\left(\frac{\alpha}{\beta}\right)_{i i}}+\frac{\gamma}{\beta}<\frac{(1-\alpha)}{\beta} \frac{\alpha}{\beta}
$$

and since $\frac{1}{R\left(\frac{\alpha}{\beta}\right)_{i i}} \leq \frac{\beta-\alpha}{\beta}$, we have

$$
\frac{(1-\alpha)^{3}}{\beta}+\frac{\gamma}{\beta}<\frac{(1-\alpha)}{\beta} \frac{\alpha}{\beta}
$$

which is equivalent to the fact that

$$
\frac{(1-\alpha)^{3}}{\beta}+\frac{\gamma}{\beta}-\frac{(1-\alpha)}{\beta} \frac{\alpha}{\beta}<0 .
$$

Again, this is a contradiction since for every $\alpha \in(0,1)$,

$$
\beta(1-\alpha)^{3}+\beta \gamma-\alpha(1-\alpha)=(1-\alpha)^{3}>0 .
$$

\section{COMPARISON OF PAGERANK INTERVALS $\mathcal{P} \mathcal{R}_{\alpha}(i)$ AND $\mathcal{P} \mathcal{R B}_{\alpha}(i)$}

In order to compare the intervals $\mathcal{P} \mathcal{R}_{\alpha}(i)$ and $\mathcal{P} \mathcal{R} \mathcal{B}_{\alpha}(i)$, we first focus on the upper limits, that is, we compare the values of $x_{i i}$ [where the upper limit of $\mathcal{P} \mathcal{R}_{\alpha}(i)$ is reached for a given $i$ ] and the values of $c_{i i}$ [where the upper limit of $\mathcal{P} \mathcal{R} \mathcal{B}_{\alpha}(i)$ is reached for the same node $i]$. We split this study into two parts, corresponding to the cases $x_{i i} \leq c_{i i}$ and $c_{i i} \leq x_{i i}$. After that, we will focus on the comparison of the lower limits of the intervals $\mathcal{P} \mathcal{R}_{\alpha}(i)$ and $\mathcal{P} \mathcal{R B}_{\alpha}(i)$ by comparing the minimum of $x_{i j}$ and $c_{i j}$ for a given $j$.

\section{A. When does $\boldsymbol{x}_{i i} \leq \boldsymbol{c}_{i i}$ hold?}

We will separate the study in two cases, according to the values of $\alpha$ :

Case 1. We will show that $x_{i i} \leq c_{i i}$ for all $\alpha \in(0.34,0.48)$. Moreover, when $M_{i}$ decreases to 0 , this interval expands to $(0.34,0.5)$.

Notice that $P_{i i}=0$ since there are no self-loops, so by using (3) and (4), $x_{i i} \leq c_{i i}$ if and only if

$$
R(\alpha)_{i i}-(1-\alpha)\left[1+\frac{\alpha(2-\alpha)}{\beta} R\left(\frac{\alpha}{\beta}\right)_{i i}\right] \leq 0 .
$$

By using the series expansion of $R(\cdot)$, the last expression is equivalent to the following:

$$
\alpha-\frac{\gamma}{\beta}+\sum_{k=2}^{\infty}\left(\alpha^{k}-\frac{\gamma}{\beta}\left(\frac{\alpha}{\beta}\right)^{k}\right)\left(P^{k}\right)_{i i} \leq 0 .
$$

Hence, if we define the function $\varphi(\alpha, k)$ for every $k \in \mathbb{N} \cup\{0\}$ and every $\alpha \in(0,1)$ by

$$
\varphi(\alpha, k)=\alpha^{k}-\frac{\gamma}{\beta}\left(\frac{\alpha}{\beta}\right)^{k},
$$

then $x_{i i} \leq c_{i i}$ if and only if

$$
\sum_{k=3}^{\infty} \varphi(\alpha, k)\left(P^{k}\right)_{i i} \leq \frac{\alpha(1-2 \alpha)}{\beta}-\varphi(\alpha, 2)\left(P^{2}\right)_{i i} .
$$


We are going to show that (9) holds when $\alpha \in(0.38,0.48)$, by analyzing the behavior of this function $\varphi(\alpha, k)$.

For $k=2, \varphi(\alpha, 2)=\alpha^{2}\left(1-\frac{\gamma}{\beta^{3}}\right)$ is always positive for all $\alpha \in$ $(0,1)$ since both $\alpha$ and $\beta^{3}-\gamma$ are positive for all $\alpha \in(0,1)$.

Notice that for $k \geq 3$, all the functions $\varphi(\alpha, k)$ are negative in the interval $(0.34,0.60)$. Indeed, since

$$
\varphi(\alpha, k)=\alpha^{k}\left(1-\frac{\gamma}{\beta^{k+1}}\right),
$$

it suffices to check that $1-\frac{\gamma}{\beta^{k+1}}<0$, for all $k \geq 3$ in $(0.34,0.60)$ or equivalently that $0<\log (\gamma)-(k+1) \log (\beta)$, which in turn is equivalent to $k+1>\frac{\log (\gamma)}{\log (\beta)}$ [notice that $\beta(x)<1$, for all $x \in(0,1)$ ], which occurs for $k \geq 3$.

Moreover, $\left(P^{2}\right)_{i i} \leq M_{i}$, where $M_{i}$ denotes the maximum entry of the $i$ th-column of $P$, so for $\alpha \in(0.34,0.60)$, we have that $x_{i i} \leq c_{i i}$ as long as

$$
\frac{\alpha(1-2 \alpha)}{\beta}-\varphi(\alpha, 2) M_{i} \geq 0,
$$

which is equivalent to

$$
\frac{\alpha(1-2 \alpha)}{\beta \varphi(\alpha, 2)} \geq M_{i}
$$

Note that this holds true in the interval $(0.34,0.48)$ because

$$
M_{i} \leq 1 \leq \frac{\alpha(1-2 \alpha)}{\beta \varphi(\alpha, 2)},
$$

for those $\alpha \in(0.34,0.48)$. Furthermore, when $M_{i}$ decreases to 0 , this interval expands to $(0.34,0.50)$ (see the top left panel in Fig. 1).

Case 2. We will show that $x_{i i} \leq c_{i i}$ for all $\alpha \in(0,0.39)$.

From (8) together with Lemma 2.5 (iii), it follows that if

$$
\frac{1-\gamma}{1-\alpha} R(\alpha)_{i i}\left[1-\frac{\gamma \alpha(1-\alpha)}{(1-\gamma) \beta} R(\alpha)_{i i}\right] \leq 1,
$$

then $x_{i i} \leq c_{i i}$.

\section{Since}

$$
\left[1-\frac{\gamma \alpha(1-\alpha)}{(1-\gamma) \beta} R(\alpha)_{i i}\right] \geq 1-\frac{\gamma \alpha(1-\alpha)}{(1-\gamma) \beta} \frac{1}{1-\alpha}>1 / 2>0,
$$

for all $\alpha \in(0,1)$ (see the top right panel in Fig. 1), then (10) holds if and only if

$$
\frac{1-\gamma}{1-\alpha} R(\alpha)_{i i} \leq\left[1-\frac{\gamma \alpha(1-\alpha)}{(1-\gamma) \beta} R(\alpha)_{i i}\right]^{-1},
$$

but if we consider the geometric series, this is equivalent to the fact that the expression

$$
\begin{gathered}
1+R(\alpha)_{i i}\left[\frac{\gamma \alpha(1-\alpha)}{(1-\gamma) \beta}-\frac{1-\gamma}{1-\alpha}\right] \\
+\sum_{k=2}\left(\frac{\gamma \alpha(1-\alpha)}{(1-\gamma) \beta}\right)^{k}\left(R(\alpha)_{i i}\right)^{k}
\end{gathered}
$$

is non-negative. Since for every $\alpha \in(0,1)$ (see the bottom right panel in Fig. 1),

$$
\left[\frac{\gamma \alpha(1-\alpha)}{(1-\gamma) \beta}-\frac{1-\gamma}{1-\alpha}\right]<0,
$$

then Lemma 2.1 implies that

$$
\begin{aligned}
& R(\alpha)_{i i}\left[\frac{\gamma \alpha(1-\alpha)}{(1-\gamma) \beta}-\frac{1-\gamma}{1-\alpha}\right] \\
& \quad \geq\left(1+M_{i} \frac{\alpha^{2}}{1-\alpha}\right)\left[\frac{\gamma \alpha(1-\alpha)}{(1-\gamma) \beta}-\frac{1-\gamma}{1-\alpha}\right] .
\end{aligned}
$$

Notice also that $R(\alpha)_{i i} \geq 1$ implies that

$$
\begin{aligned}
\frac{\frac{\gamma^{2} \alpha^{2}(1-\alpha)^{2}}{(1-\gamma)^{2} \beta^{2}}}{1-\frac{\gamma \alpha(1-\alpha)}{(1-\gamma) \beta}} & =\sum_{k=2}\left(\frac{\gamma \alpha(1-\alpha)}{(1-\gamma) \beta}\right)^{k} \\
& \leq \sum_{k=2}\left(\frac{\gamma \alpha(1-\alpha)}{(1-\gamma) \beta}\right)^{k}\left(R(\alpha)_{i i}\right)^{k} .
\end{aligned}
$$

Consequently,

$$
\begin{aligned}
\Phi\left(\alpha, M_{i}\right) \leq & 1+R(\alpha)_{i i}\left[\frac{\gamma \alpha(1-\alpha)}{(1-\gamma) \beta}-\frac{1-\gamma}{1-\alpha}\right] \\
& +\sum_{k=2}\left(\frac{\gamma \alpha(1-\alpha)}{(1-\gamma) \beta}\right)^{k}\left(R(\alpha)_{i i}\right)^{k},
\end{aligned}
$$

where $\Phi\left(\alpha, M_{i}\right)$ is defined as

$$
\begin{aligned}
\Phi\left(\alpha, M_{i}\right)=1 & +\left(1+M_{i} \frac{\alpha^{2}}{1-\alpha}\right)\left[\frac{\gamma \alpha(1-\alpha)}{(1-\gamma) \beta}-\frac{1-\gamma}{1-\alpha}\right] \\
& +\frac{\frac{\gamma^{2} \alpha^{2}(1-\alpha)^{2}}{(1-\gamma)^{2} \beta^{2}}}{1-\frac{\gamma \alpha(1-\alpha)}{(1-\gamma) \beta}},
\end{aligned}
$$

and thus, we have that $x_{i i} \leq c_{i i}$ as long as $\Phi\left(\alpha, M_{i}\right) \geq 0$ (see the left panel in Fig. 2).

Notice that the fact that

$$
R(\alpha)_{i i}<\frac{1}{1-\alpha}
$$

was used to get

$$
\frac{\gamma \alpha(1-\alpha)}{(1-\gamma) \beta} R(\alpha)_{i i}<\frac{\gamma \alpha(1-\alpha)}{(1-\gamma) \beta} \frac{1}{1-\alpha},
$$

which satisfies that

$$
\frac{\gamma \alpha(1-\alpha)}{(1-\gamma) \beta} \frac{1}{1-\alpha}<1
$$

for all $\alpha \in(0,1)$. Observe that the sign of the function $\Phi\left(\alpha, M_{i}\right)$ depends on $M_{i}$. In fact, $\Phi\left(\alpha, M_{i}\right)=0$ if and only if

$$
M_{i}=-\left(\frac{1+R_{2}(\alpha)}{\frac{\gamma \alpha(1-\alpha)}{(1-\gamma) \beta}-\frac{1-\gamma}{1-\alpha}}+1\right) \frac{1-\alpha}{\alpha^{2}},
$$



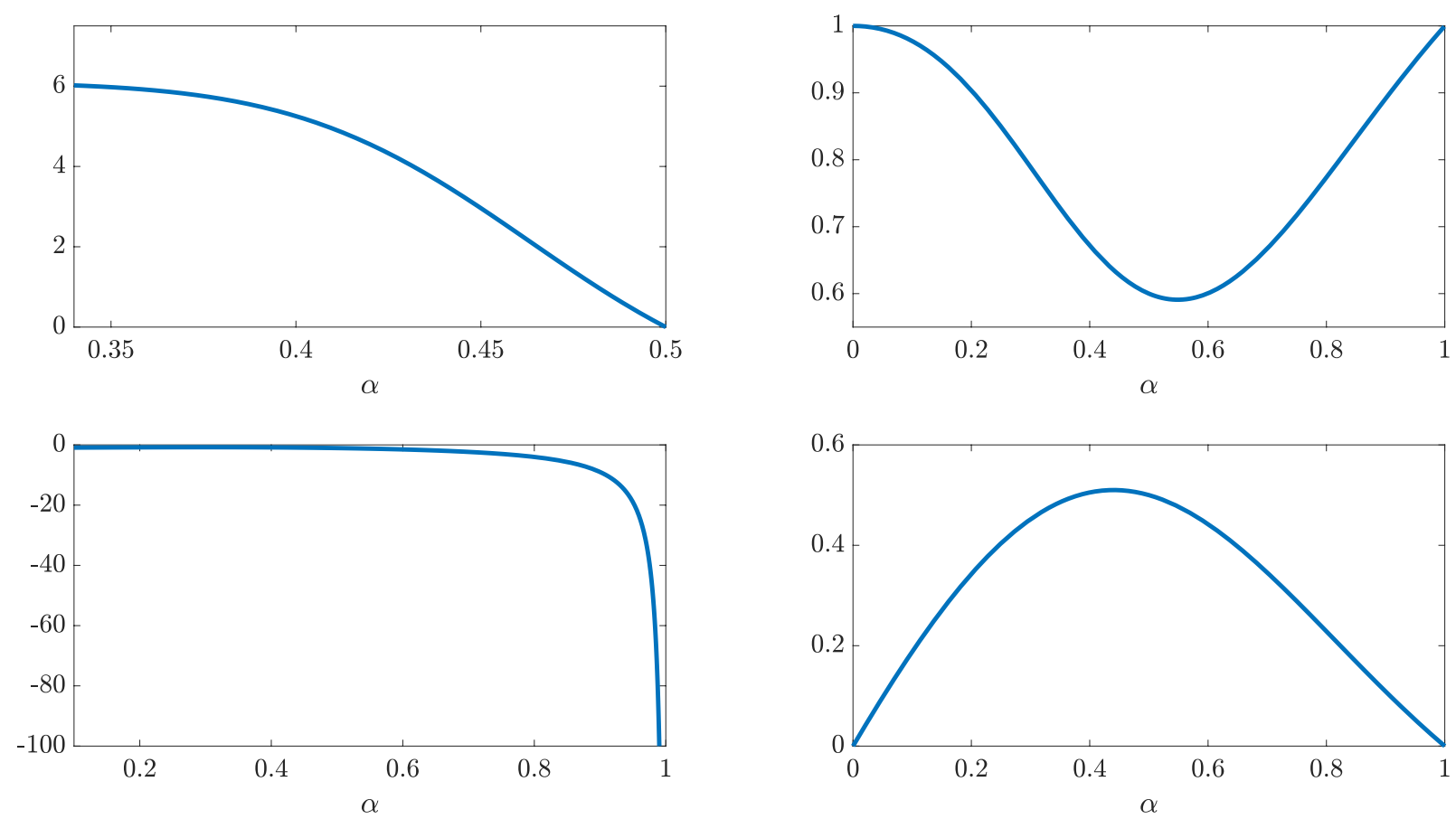

FIG. 1. Some auxiliary functions: $\frac{\alpha(1-2 \alpha)}{\beta \varphi(\alpha, 2)}$ in $(0.34,0.48)$ (top left panel), $1-\frac{\gamma \alpha(1-\alpha)}{(1-\gamma) \beta} \frac{1}{1-\alpha}$ (top right panel), $\frac{\gamma \alpha(1-\alpha)}{(1-\gamma) \beta}-\frac{1-\gamma}{1-\alpha}$ (bottom left panel), and function $\frac{\gamma}{\beta}$ (bottom right panel).

where

$$
R_{2}(\alpha)=\frac{\frac{\gamma^{2} \alpha^{2}(1-\alpha)^{2}}{(1-\gamma)^{2} \beta^{2}}}{1-\frac{\gamma \alpha(1-\alpha)}{(1-\gamma) \beta}}
$$

but the function

$$
\Psi(\alpha)=-\left(\frac{1+R_{2}(\alpha)}{\frac{\gamma \alpha(1-\alpha)}{(1-\gamma) \beta}-\frac{1-\gamma}{1-\alpha}}+1\right) \frac{1-\alpha}{\alpha^{2}}
$$

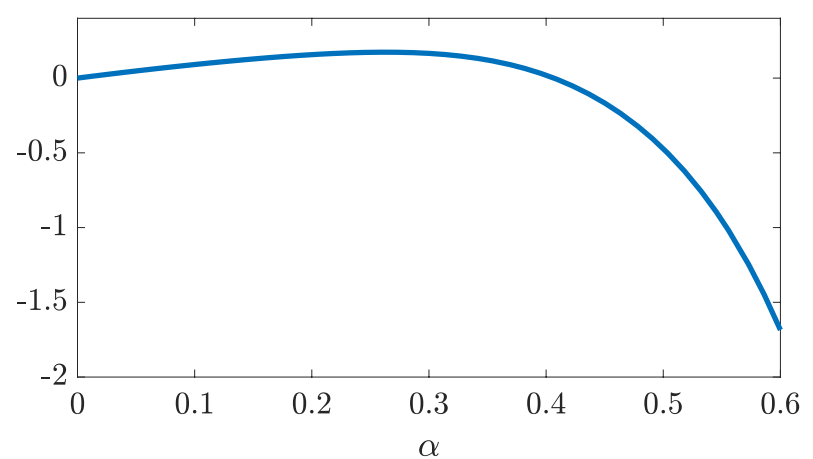

can be seen to take values in $[0,1]$ only for $\alpha \in[\xi, 0.5]$ with $\xi \approx 0.399$ (see the right panel in Fig. 2).

This means that $\Phi\left(\alpha, M_{i}\right)=0$ has a single root $\alpha_{i}$ in $[0,1]$, which must necessarily lie in $[\xi, 0.5]$. In addition, $\Phi\left(\alpha, M_{i}\right)$ takes positive values on the interval $\left(0, \alpha_{i}\right)$ and negative ones on the interval $\left(\alpha_{i}, 1\right]$. It follows that $\Phi\left(\alpha, M_{i}\right) \geq 0$ for every $\alpha \in(0, \xi]$ and every $i$; consequently, $x_{i i} \leq c_{i i}$, for all $\alpha \in(0, \xi]$.

Notice that the intervals obtained in Case 1 and in Case 2 overlap and lead the conclusion that $x_{i i} \leq c_{i i}$, for all $\alpha \in(0,0.48)$. Moreover, this interval expands to $(0,0.5)$ as $M_{i}$ decreases to 0 , that

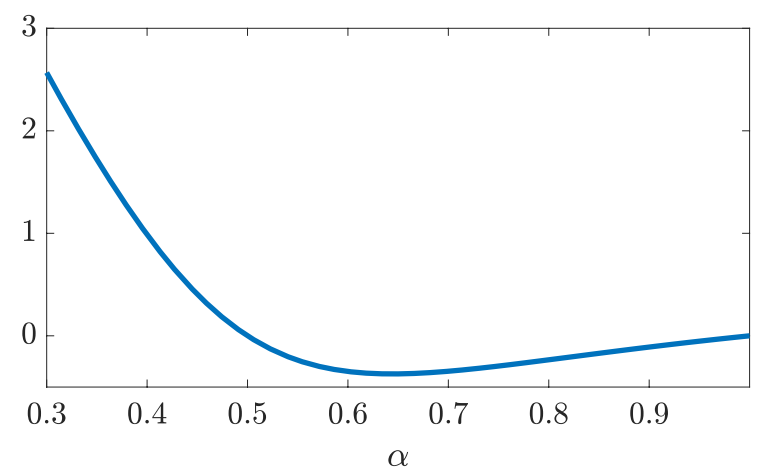

FIG. 2. More auxiliary functions: $\Phi(\alpha, 0.9)$ (left side) and $\Psi$ (right side). 
is, the inequality $x_{i i} \leq c_{i i}$ also holds for $\alpha \in[0.48,0.5)$ for $M_{i}$ small enough.

\section{B. When does $c_{i i} \leq x_{i i}$ hold?}

We will define two intervals $I_{\lambda}$ and $H_{\lambda, M_{i}}$ depending on a certain parameter $\lambda \in(0,1)$ and on the maximum value $M_{i}$ of the entries of the $i$ th-column of $P$ such that for all $\alpha \in I_{\lambda} \cap H_{\lambda, M_{i}}$, we will have that $c_{i i} \leq x_{i i}$.

We make the important remark that the approach previously taken for the inequality $x_{i i} \leq c_{i i}$ involved the Resolvent Identity (6) together with some estimation given in Lemma 2.5 (iii). Unfortunately, in our present situation, such an approach turns out to be unsuccessful. The reason is that the estimation in Lemma 2.5 gives now irrelevant information. that

Thus, a different approach must be taken. From (8), it follows

$$
\begin{aligned}
\frac{(C-X)_{i i}}{(1-\alpha)^{2}} & =1-\frac{1-\gamma}{1-\alpha} R(\alpha)_{i i}+\frac{\gamma \alpha(1-\alpha)}{\beta(1-\alpha)}\left(R\left(\frac{\alpha}{\beta}\right) R(\alpha)\right)_{i i} \\
& =1-\frac{1-\gamma}{1-\alpha} R(\alpha)_{i i}+\frac{\alpha \gamma}{\beta}\left(R\left(\frac{\alpha}{\beta}\right) R(\alpha)\right)_{i i},
\end{aligned}
$$

so $c_{i i} \leq x_{i i}$ if and only if

$$
1-\frac{1-\gamma}{1-\alpha} R(\alpha)_{i i}+\frac{\alpha \gamma}{\beta}\left(R\left(\frac{\alpha}{\beta}\right) R(\alpha)\right)_{i i} \leq 0 .
$$

For $0<\lambda<1$, consider $\eta_{\lambda}(\alpha)=\alpha^{\lambda}-\frac{\alpha}{\beta}$ and

$$
I_{\lambda}=\left\{\alpha \in[0,1]: \eta_{\lambda}(\alpha) \geq 0\right\} .
$$

Notice that $I_{\lambda}$ expands to $[0,1]$ as $\lambda$ decreases to 0 and narrows down to the two-point set $\{0,1\}$ as $\lambda$ increases to 1 (see Fig. 3 ).

Note that if $\alpha \in I_{\lambda}$ and

$$
1-\frac{1-\gamma}{1-\alpha} R(\alpha)_{i i}+\frac{\alpha \gamma}{\beta}\left(R\left(\alpha^{\lambda}\right) R(\alpha)\right)_{i i} \leq 0,
$$

then $c_{i i} \leq x_{i i}$, since by $(6)$

$$
\begin{aligned}
R\left(\alpha^{\lambda}\right) R(\alpha) & =\frac{1}{\alpha-\alpha^{\lambda}}\left(\alpha R(\alpha)-\alpha^{\lambda} R\left(\alpha^{\lambda}\right)\right) \\
& =\frac{1}{1-\alpha^{\lambda-1}}\left[R(\alpha)-\alpha^{\lambda-1} \sum_{k=0}^{\infty}\left(\alpha^{\lambda}\right)^{k} P^{k}\right],
\end{aligned}
$$

so $\left(R\left(\alpha^{\lambda}\right) R(\alpha)\right)_{i i}$ can be written as

$$
\frac{1}{1-\alpha^{\lambda-1}}\left[R(\alpha)_{i i}-\alpha^{\lambda-1} \sum_{k=0}^{\infty}\left(\alpha^{\lambda}\right)^{k}\left(P^{k}\right)_{i i}\right] .
$$

Thus, for $\alpha \in I_{\lambda}$, the condition

$$
\begin{aligned}
1- & \frac{1-\gamma}{1-\alpha} R(\alpha)_{i i}+\frac{\alpha \gamma}{\beta} \frac{1}{1-\alpha^{\lambda-1}} \\
& {\left[R(\alpha)_{i i}-\alpha^{\lambda-1}\left(1+\sum_{k=2}^{\infty}\left(\alpha^{\lambda}\right)^{k}\left(P^{k}\right)_{i i}\right)\right] \leq 0, }
\end{aligned}
$$

would imply that $c_{i i} \leq x_{i i}$, and this condition is equivalent to

$$
\begin{aligned}
1+ & {\left[\frac{\alpha \gamma}{\beta} \frac{1}{1-\alpha^{\lambda-1}}-\frac{1-\gamma}{1-\alpha}\right] R(\alpha)_{i i} } \\
& -\frac{\gamma}{\beta} \frac{\alpha^{\lambda}}{1-\alpha^{\lambda-1}}\left(1+\sum_{k=2}^{\infty}\left(\alpha^{\lambda}\right)^{k}\left(P^{k}\right)_{i i}\right) \leq 0 .
\end{aligned}
$$

Notice that for all $\alpha \in(0,1)$, we have that

$$
\frac{\gamma}{\beta} \frac{\alpha^{\lambda-1}}{1-\alpha^{\lambda-1}}<0
$$

and $\left(P^{k}\right)_{i i} \leq M_{i}$ for all $k \geq 2$. Also,

$$
\left[\frac{\alpha \gamma}{\beta} \frac{1}{1-\alpha^{\lambda-1}}-\frac{1-\gamma}{1-\alpha}\right]<0,
$$

and $1 \leq R(\alpha)_{i i}$, so the following sufficient condition is obtained: if $\alpha \in I_{\lambda} \cap H_{\lambda, M_{i}}$, then $c_{i i} \leq x_{i i}$, where $H_{\lambda, M_{i}}$ is defined as

$$
H_{\lambda, M_{i}}=\left\{\alpha \in(0,1): \theta\left(\alpha, \lambda, M_{i}\right) \leq 0\right\},
$$

where $\theta\left(\alpha, \lambda, M_{i}\right)$ is given by

$$
1+\left[\frac{\alpha \gamma}{\beta} \frac{1}{1-\alpha^{\lambda-1}}-\frac{1-\gamma}{1-\alpha}\right]-\frac{\gamma}{\beta} \frac{\alpha^{\lambda}}{1-\alpha^{\lambda-1}}\left[1+\frac{M_{i} \alpha^{2 \lambda}}{1-\alpha^{\lambda}}\right] .
$$

Notice that $\theta\left(\alpha, \lambda, M_{i}\right)$ takes positive values for all $\alpha \in(0,0.5], \lambda \in$ $(0,1)$, and $M_{i} \in(0,1]$.

As mentioned above, $I_{\lambda}$ expands from right to left to $(0,1)$ as $\lambda$ decreases to 0 . If $M_{i}$ is taken sufficiently smaller than $\lambda$, then $H_{\lambda, M_{i}}$ is not empty and expands to $[0.5,1)$ as $M_{i}$ decreases to 0 (see Fig. 4 ).

Notice that $[0.5,1)$ contains all $\alpha$ 's for which the upper extreme of the Biplex PageRank is smaller than the upper extreme of the Classical PageRank; particularly, as $M_{i}$ gets closer to zero, the interval of such $\alpha$ 's exhausts the interval $[0.5,1)$. For instance, for $\lambda=0.15$
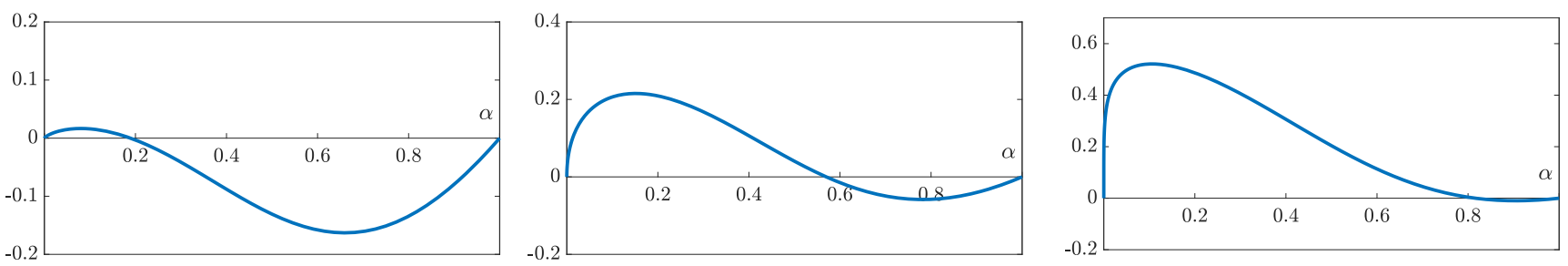

FIG. 3. $I_{\lambda}$ for different $\lambda$ 's: $\eta_{0.9}$ and $I_{0.9}$ (left panel), $\eta_{0.5}$ and $I_{0.5}$ (center panel), and $\eta_{0.2}$ and $I_{0.2}$ (right panel). 

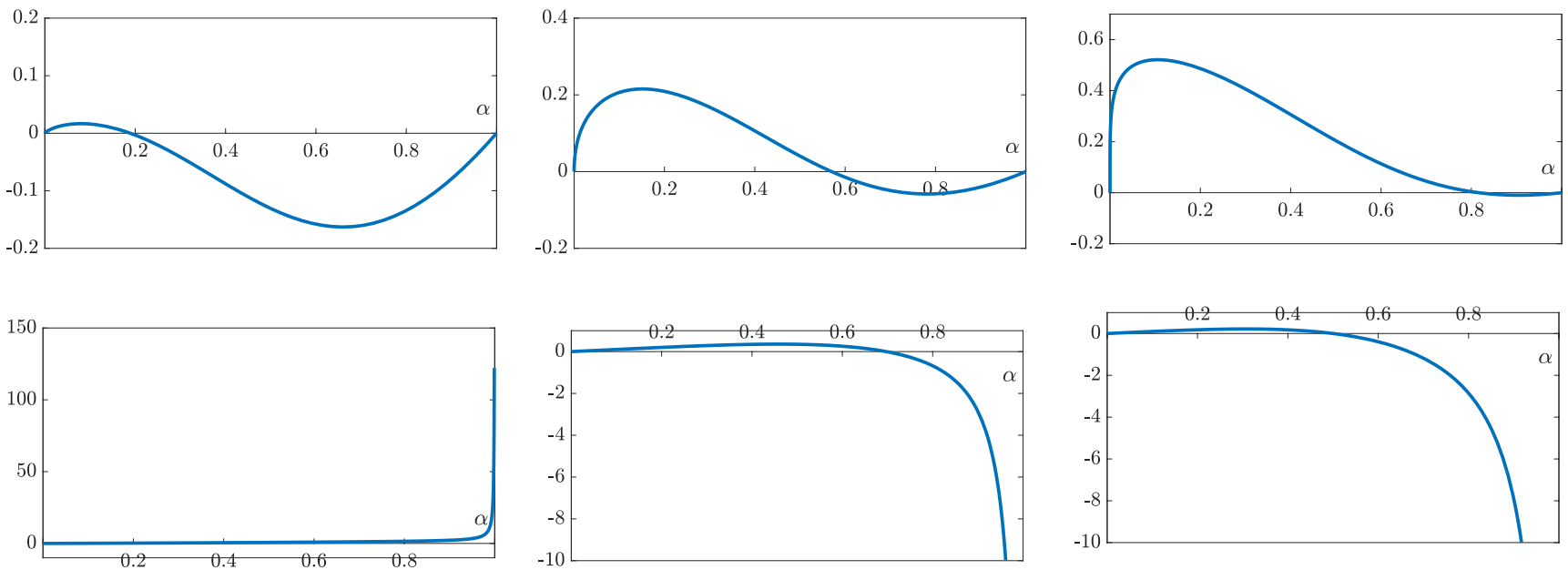

FIG. 4. $I_{0.1}$ and $H_{0.1, M_{i}}$ for different $M_{i}$ 's: $\eta_{0.9}$ and $I_{0.9}$ (top left panel), $\eta_{0.5}$ and $I_{0.5}$ (top central panel), $\eta_{0.2}$ and $I_{0.2}$ (top right panel), $\varphi(\alpha, 0.1,0.1)$ (bottom left panel), $\varphi(\alpha, 0.1,0.05)$ (bottom central panel), and $\varphi(\alpha, 0.1,0.0001)$ (bottom right panel).

and $M_{i}=0.1$, the value $\alpha=0.85$ falls into $H_{0.15,0.10}$. This means that for $\alpha=0.85$, we obtain $c_{i i} \leq x_{i i}$ when the maximum value of the $i$ th-column of $P$ is less than or equal to 0.1 .

\section{Comparison of the lower extremes of the intervals}

Now, we turn to the lower extremes of the Classical PageRank and the Biplex PageRank intervals. In this case, we will only obtain that $\min _{i} c_{i j} \leq \min _{i} x_{i j}$ for "small" $\alpha$ 's.

We make the trivial observation that $R\left(\frac{\alpha}{\beta}\right)_{i j}=0$ automatically implies $0=R(\alpha)_{i j}=\min _{i} x_{i j}=\min _{i} c_{i j}$. On the other hand, $R(\alpha)_{i j}=0$ implies $\min _{i} x_{i j} \leq \min _{i} c_{i j}$. Thus, we will assume in what follows $\min _{i} R(\alpha)_{i j}>0$.

Notice that by Corollary 2.4, it can be safely assumed that

$$
\min _{i} x_{i j}=\min _{i \neq j} x_{i j} \quad \text { and } \quad \min _{i} c_{i j}=\min _{i \neq j} c_{i j} \text {. }
$$

Recall that for $i \neq j$, (4) and (3) become

$$
x_{i j}=(1-\alpha) R(\alpha)_{i j} \quad \text { and } \quad c_{i j}=\frac{(1-\alpha) \gamma}{\beta} R\left(\frac{\alpha}{\beta}\right)_{i j},
$$

so it readily follows

$$
\min _{i \neq j} x_{i j} \geq \min _{i \neq j} c_{i j} \Longleftrightarrow \frac{\min _{i \neq j} R(\alpha)_{i j}}{\min _{i \neq j} R\left(\frac{\alpha}{\beta}\right)_{i j}} \geq \frac{\gamma}{\beta} .
$$

For $i \neq j$, let us calculate

$$
\lim _{\alpha \rightarrow 0} \frac{R(\alpha)_{i j}}{R\left(\frac{\alpha}{\beta}\right)_{i j}}
$$

From

$$
0 \leq R(\alpha)_{i j} \leq R(\alpha)_{j j} \leq \frac{\alpha}{1-\alpha}
$$

and

$$
0 \leq R\left(\frac{\alpha}{\beta}\right)_{i j} \leq R\left(\frac{\alpha}{\beta}\right)_{j j} \leq \frac{\alpha}{\beta-\alpha},
$$

it follows that

$$
\lim _{\alpha \rightarrow 0} R\left(\frac{\alpha}{\beta}\right)_{i j}=\lim _{\alpha \rightarrow 0} R(\alpha)_{i j}=0 .
$$

In addition to this,

$$
\frac{d}{d \alpha} R(\alpha)_{i j}=\sum_{k=1} k \alpha^{k-1}\left(P^{k}\right)_{i j}
$$

and

$$
\frac{d}{d \alpha} R\left(\frac{\alpha}{\beta}\right)_{i j}=\frac{1-\alpha^{2}}{\beta^{2}} \sum_{k=1} k\left(\frac{\alpha}{\beta}\right)^{k-1}\left(P^{k}\right)_{i j},
$$

which makes

$$
\lim _{\alpha \rightarrow 0} \frac{d}{d \alpha} R(\alpha)_{i j}=P_{i j}=\lim _{\alpha \rightarrow 0} \frac{d}{d \alpha} R\left(\frac{\alpha}{\beta}\right)_{i j} .
$$

It suffices now to use L'Hôpital's rule to get that

$$
\lim _{\alpha \rightarrow 0} \frac{R(\alpha)_{i j}}{R\left(\frac{\alpha}{\beta}\right)_{i j}}=1 .
$$

Finally, notice that $\gamma / \beta<0.6$ for all $\alpha \in(0,1)$ evidently implies that for $\alpha$ close to zero,

$$
\frac{\min _{i \neq j} R(\alpha)_{i j}}{\min _{i \neq j} R\left(\frac{\alpha}{\beta}\right)_{i j}} \geq \frac{\gamma}{\beta},
$$

which is equivalent to

$$
\min _{i} x_{i j} \geq \min _{i} c_{i j}
$$




\section{SOME EXAMPLES AND SIMULATIONS}

In this final section, an analytical example, some simulations on synthetic random models and real examples are included in order to illustrate the results obtained in Secs. II and III.

Given a complex network of $n$ nodes, $i \in\{1, \ldots, n\}$ and $\alpha \in$ $(0,1)$, if we use Theorem 2.6 , then the possible relative position of intervals $\mathcal{P} \mathcal{R}_{\alpha}(i)=\left(x_{\min }, x_{\max }\right)$ and $\mathcal{P} \mathcal{R} \mathcal{B}_{\alpha}(i)=\left(c_{\min }, c_{\max }\right)$ can be one of the following:

- Type 1: if $\mathcal{P} \mathcal{R}_{\alpha}(i) \subseteq \mathcal{P} \mathcal{R} \mathcal{B}_{\alpha}(i)$, i.e.,

$$
c_{\min } \leq x_{\min }<x_{\max } \leq c_{\max }
$$

- Type 2: if $x_{\min }<c_{\min }<x_{\max } \leq c_{\max }$,

- Type 3: if $c_{\min } \leq x_{\min }<c_{\max }<x_{\max }$, and

- Type 4: if $\mathcal{P} \mathcal{R} \mathcal{B}_{\alpha}(i) \subset \mathcal{P} \mathcal{R}_{\alpha}($ i), i.e.,

$$
x_{\min }<c_{\min }<c_{\max }<x_{\max } .
$$

Following this notation, we are going to compute which nodes (or how many of them) have a relative position of intervals $\mathcal{P} \mathcal{R}_{\alpha}(i)$ and $\mathcal{P} \mathcal{R} \mathcal{B}_{\alpha}(i)$ of Types $1,2,3$, and 4 . First, we consider an example where each interval $\mathcal{P} \mathcal{R}_{\alpha}(i)$ and $\mathcal{P} \mathcal{R} \mathcal{B}_{\alpha}(i)$ can be analytically computed.

Example 4.1. If we consider $G=S_{n}$ the undirected star of $n+1$ nodes with center at node 1 , then the row-stochastic matrix $P$ associated with $S_{n}$ is

$$
P=\left(\begin{array}{c|ccc}
0 & \frac{1}{n} & \ldots & \frac{1}{n} \\
\hline 1 & 0 & & 0 \\
\vdots & \vdots & \ddots & \vdots \\
1 & 0 & \ldots & 0
\end{array}\right) .
$$

It is easy to check that $P^{0}=I$ and for every $0 \leq k \in \mathbb{N} \cup\{0\}$, we get that

$$
\begin{aligned}
P^{2 k+1} & =\left(\begin{array}{c|ccc}
0 & \frac{1}{n} & \ldots & \frac{1}{n} \\
\hline 1 & 0 & \ldots & 0 \\
\vdots & \vdots & \ddots & \vdots \\
1 & 0 & \ldots & 0
\end{array}\right)=\mathbb{M}_{1}, \\
P^{2 k+2} & =\left(\begin{array}{c|ccc}
1 & 0 & \ldots & 0 \\
\hline 0 & \frac{1}{n} & \ldots & \frac{1}{n} \\
\vdots & \vdots & \ddots & \vdots \\
0 & \frac{1}{n} & \cdots & \frac{1}{n}
\end{array}\right)=\mathbb{M}_{2} .
\end{aligned}
$$

Therefore, by using some properties of the geometric series, for every $\alpha \in(0,1)$,

$$
\begin{aligned}
R(\alpha) & =I+\sum_{k=0}^{\infty} \alpha^{2 k+1} P^{2 k+1}+\sum_{k=0}^{\infty} \alpha^{2 k+2} P^{2 k+2} \\
& =I+\frac{\alpha}{1-\alpha^{2}} \mathbb{M}_{1}+\frac{\alpha^{2}}{1-\alpha^{2}} \mathbb{M}_{2}, \\
R\left(\frac{\alpha}{\beta}\right) & =I+\frac{\alpha \beta}{\beta^{2}-\alpha^{2}} \mathbb{M}_{1}+\frac{\alpha^{2}}{\beta^{2}-\alpha^{2}} \mathbb{M}_{2} .
\end{aligned}
$$

Hence, by using (3) and (4),

$$
\begin{aligned}
X & =(1-\alpha) R(\alpha)=(1-\alpha) I+\frac{\alpha}{1+\alpha} \mathbb{M}_{1}+\frac{\alpha^{2}}{1+\alpha} \mathbb{M}_{2}, \\
C & =(1-\alpha)^{2}\left(I+\frac{\alpha(2-\alpha)}{\beta} R\left(\frac{\alpha}{\beta}\right)\right) \\
& =\frac{(1-\alpha)^{2}(1+\alpha)}{\beta} I+\frac{\alpha^{2}(2-\alpha)}{1+\alpha^{2}} \mathbb{M}_{1}+\frac{\alpha^{3}(2-\alpha)}{\beta\left(1+\alpha^{2}\right)} \mathbb{M}_{2} .
\end{aligned}
$$

By using these expressions of $X$ and $C$, if we calculate $x_{11}$ and $c_{11}$, we get that

$$
\begin{aligned}
& x_{11}=(1-\alpha)+\frac{\alpha^{2}}{1+\alpha}, \\
& c_{11}=\frac{(1-\alpha)^{2}(1+\alpha)}{\beta}+\frac{\alpha^{3}(2-\alpha)}{\beta\left(1+\alpha^{2}\right)},
\end{aligned}
$$

and, therefore, by direct computation, we obtain that

$$
x_{11}<c_{11} \Longleftrightarrow \alpha \in\left(0, \frac{\sqrt{5}-1}{2}\right) .
$$

Now let us compare $x_{i i}$ with $c_{i i}$ for $2 \leq i \leq n$. Note that, in this case,

$$
\begin{aligned}
& x_{i i}=(1-\alpha)+\frac{1}{n} \frac{\alpha^{2}}{1+\alpha}, \\
& c_{i i}=\frac{(1-\alpha)^{2}(1+\alpha)}{\beta}+\frac{1}{n} \frac{\alpha^{3}(2-\alpha)}{\beta\left(1+\alpha^{2}\right)},
\end{aligned}
$$

and hence,

$$
x_{i i}<c_{i i} \Longleftrightarrow \alpha \in\left(0, \xi_{n}\right),
$$

with $\xi_{n}>0.5$ for all $n \geq 1$ and $\xi_{n} \longrightarrow 0.5^{+}$, as $n$ increases to infinity. Let us compare $x_{j 1}$ with $c_{j 1}$, for every $1 \leq j \leq n$. Note that

$$
\begin{aligned}
& x_{j 1}=\frac{\alpha}{1+\alpha}, \\
& c_{j 1}=\frac{\alpha^{2}(2-\alpha)}{1+\alpha^{2}},
\end{aligned}
$$

and, therefore,

$$
x_{j 1}<c_{j 1} \Longleftrightarrow \alpha \in\left(\frac{\sqrt{5}-1}{2}, 1\right) .
$$

Therefore, by using (11) and (13), we get that the relative position of $\mathcal{P} \mathcal{R}_{\alpha}(1)$ and $\mathcal{P} \mathcal{R} \mathcal{B}_{\alpha}(1)$ as

- Type 1 if and only if $\alpha \in\left(0, \frac{1}{\Phi}\right]$ and

- Type 4 if and only if $\alpha \in\left(\frac{1}{\Phi}, 1\right)$,

where $\Phi$ denotes the Golden ratio. 

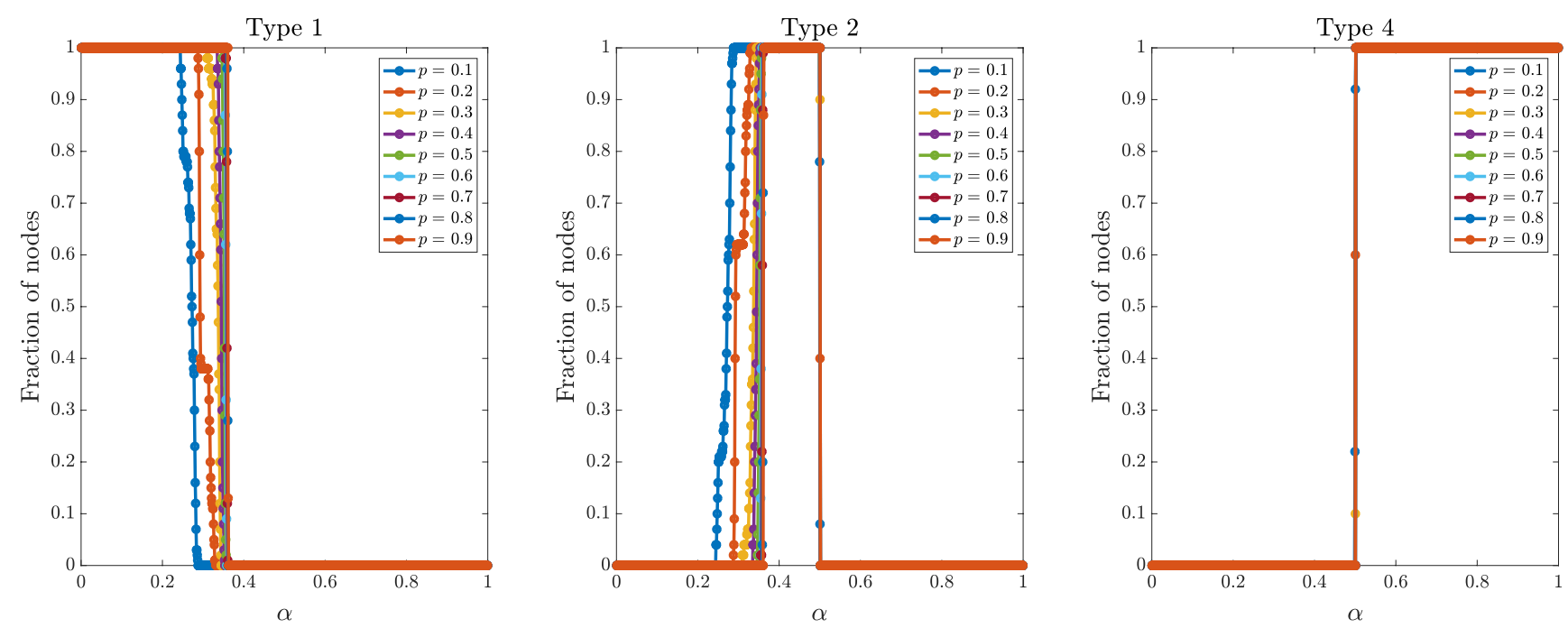

FIG. 5. Fraction of nodes in Erd"os-Rényi networks with $n=100$ nodes such that $\mathcal{P} \mathcal{R}_{\alpha}(i) \subseteq \mathcal{P} \mathcal{R} \mathcal{B}_{\alpha}$ (i) (Type 1, left panel), $x_{\min }<c_{\min } \leq x_{\max } \leq c_{\max }$ (Type 2 , central panel) and $\mathcal{P} \mathcal{R} \mathcal{B}_{\alpha}(i) \subset \mathcal{P} \mathcal{R}_{\alpha}(i)$ (Type 4, right panel) for different values of the linking probability $p$.

Finally, in order to compare $x_{j i}$ with $c_{j i}$, if $j \neq 1 \neq i$, then

$$
\begin{aligned}
x_{j i} & =\frac{1}{n} \frac{\alpha^{2}}{1+\alpha}, \\
c_{j i} & =\frac{1}{n} \frac{\alpha^{3}(2-\alpha)}{\beta\left(1+\alpha^{2}\right)},
\end{aligned}
$$

which makes that for every $j \neq 1 \neq i$,

$$
x_{j i}<c_{j i} \Longleftrightarrow \alpha \in(\eta, 1) \text {, }
$$

for

$$
\eta=\frac{1}{3}\left(-5 \sqrt[3]{\frac{2}{9 \sqrt{29}+43}}+\sqrt[3]{\frac{9 \sqrt{29}+43}{2}}-1\right) .
$$

Notice that the case $i=1 \neq j$ has no interest since we are looking for the minimum value of each column of $X$ and $C$, and it never occurs in the first row $\left(x_{j i}<x_{1 i}\right.$ and $c_{j i}<c_{1 i}$ if $\left.j \neq 1\right)$.

Hence, by using (12) and (14), if $2 \leq i \leq n$, then the relative position of $\mathcal{P} \mathcal{R}_{\alpha}(i)$ and $\mathcal{P} \mathcal{R} \mathcal{B}_{\alpha}(i)$ is

- Type 1 if and only if $\alpha \in(0, \eta]$,

- Type 2 if and only if $\alpha \in\left(\eta, \xi_{n}\right]$, and

- Type 4 if and only if $\alpha \in\left(\xi_{n}, 1\right)$,

since $\eta \approx 0.3926$ and $\xi_{n}>0.5$ for all $n \geq 1$.

Note that for every $\alpha \in(0,1)$, there is no node $i \in\{1, \ldots, n\}$ such that the relative positions of intervals $\mathcal{P} \mathcal{R}_{\alpha}(i)$ and $\mathcal{P} \mathcal{R} \mathcal{B}_{\alpha}(i)$ is of Type 3.

Now, we are going to compute the relative positions of intervals $\mathcal{P} \mathcal{R}_{\alpha}(i)$ and $\mathcal{P} \mathcal{R} \mathcal{B}_{\alpha}(i)$ for some classic synthetic random models.

Example 4.2. If we consider Erd"os-Rényi random networks with $n=100$ nodes and compute the relative position of intervals $\mathcal{P} \mathcal{R}_{\alpha}(i)$ and $\mathcal{P} \mathcal{R} \mathcal{B}_{\alpha}(i)$ for different values of the linking probability $p$ from 0.1 to 0.9 , Fig. 5 shows that for small values of $\alpha$ not bigger than $\alpha_{0}(p)$ between 0.2 and 0.4 (depending on the linking probability $p$ ) all nodes have a relative position of intervals $\mathcal{P} \mathcal{R}_{\alpha}(i)$ and $\mathcal{P} \mathcal{R} \mathcal{B}_{\alpha}(i)$ of Type 1, while if $\alpha \geq 0.5$ (independently of the linking probability $p$ ) all nodes have a relative position of intervals of Type 4 . If we take values of $\alpha$ between $\alpha_{0}(p)$ and 0.5 then there are simultaneously nodes with relative positions of intervals of Types 1 and 2, but again there are no nodes with relative position of intervals of Type 3.

Example 4.3. If we consider Barabási-Albert synthetic networks ${ }^{6}$ with $n=100$ nodes and compute the relative position of intervals $\mathcal{P R}_{\alpha}(i)$ and $\mathcal{P} \mathcal{R} \mathcal{B}_{\alpha}(i)$ for different values of the minimum degree value $d$ from 3 to 24, Fig. 6 shows that, as it happened for Erd"os-Rényi random networks, for small values of $\alpha$ not bigger than $\alpha_{0}(d)$ between 0.2 and 0.4 (depending on the minimum degree value d), all nodes have a relative position of intervals $\mathcal{P} \mathcal{R}_{\alpha}(i)$ and $\mathcal{P} \mathcal{R B}_{\alpha}(i)$ of Type 1. On the other hand, if $\alpha \geq 0.5$ (independently of the minimum degree value d) all nodes have a relative position of intervals of Type 4. If we take values of $\alpha$ between $\alpha_{0}(d)$ and 0.5 , then there are simultaneously nodes with relative positions of intervals of Types 1 and 2, but again there are no nodes with relative position of intervals of Type 3 .

Example 4.4. If we consider Watts-Strogatz synthetic networks with $n=100$ nodes (rewiring probability $\beta$ and $500 \beta$ links) and compute the relative position of intervals $\mathcal{P} \mathcal{R}_{\alpha}(i)$ and $\mathcal{P} \mathcal{R} \mathcal{B}_{\alpha}(i)$ for different values of the rewiring probability $\beta$ from 0.1 to 0.9 , Fig. 7 shows that, as it happened for Erd"os-Rényi random networks and Barabási-Albert synthetic networks, for small values of $\alpha$ not bigger than $\alpha_{0}(\beta)$ smaller than 0.4 (depending on the rewiring probability $\beta$ ) all nodes have a relative position of intervals $\mathcal{P} \mathcal{R}_{\alpha}(i)$ and $\mathcal{P} \mathcal{R} \mathcal{B}_{\alpha}(i)$ of Type 1. Again, if $\alpha \geq \alpha_{1}(\beta)$ (depending on the rewiring probability $\beta$, but close to 0.5 ), all nodes have a relative position of intervals of Type 4. Finally, if we take values of $\alpha$ between $\alpha_{0}(\beta)$ and $\alpha_{1}(\beta)$, then there are simultaneously nodes with relative positions of intervals of 

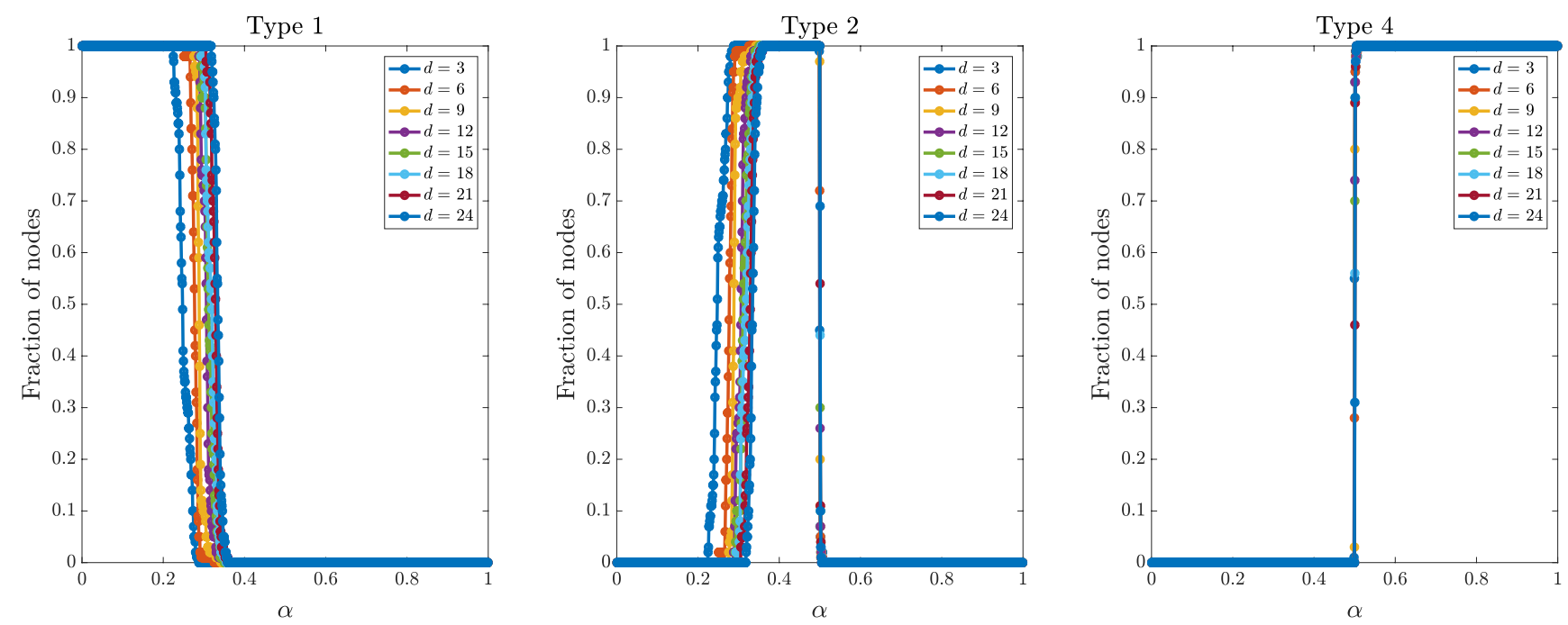

FIG. 6. Fraction of nodes in Barabási-Albert synthetic networks with $n=100$ nodes such that $\mathcal{P} \mathcal{R}_{\alpha}(i) \subseteq \mathcal{P} \mathcal{R} \mathcal{B}_{\alpha}(i)$ (Type 1, left panel), $x_{\min }<c_{\min } \leq x_{\max } \leq c_{\max }($ Type 2, central panel) and $\mathcal{P} \mathcal{R} \mathcal{B}_{\alpha}(i) \subset \mathcal{P} \mathcal{R}_{\alpha}(i)$ (Type 4, right panel) for different values of the minimum degree value $d$.

Types 1 and 2, but again there are no nodes with relative position of intervals of Type 3. There are several clear similarities of the behavior of Watts-Strogatz networks with the preceding synthetic models, but main differences with the previous synthetic examples are the following:

- Not all the nodes have relative position of intervals of Type 4 for all values of $\alpha>0.5$, so the threshold value $\alpha=0.5$ is not universal for all the networks.
- There are small values of $\alpha$ (much smaller than 0.2 ) such that there are simultaneously nodes with relative positions of intervals of Types 1 and 2.

Finally, we present an example of the relative position of intervals $\mathcal{P} \mathcal{R}_{\alpha}(i)$ and $\mathcal{P} \mathcal{R} \mathcal{B}_{\alpha}(i)$ for a real social network.

Example 4.5. We finally consider the 167 nodes giant connected component of Facebook users network presented in Ref. 43 (corresponding to the giant component of matrix called 0 in the Facebook circles network collected in Ref. 63) that consists of circles
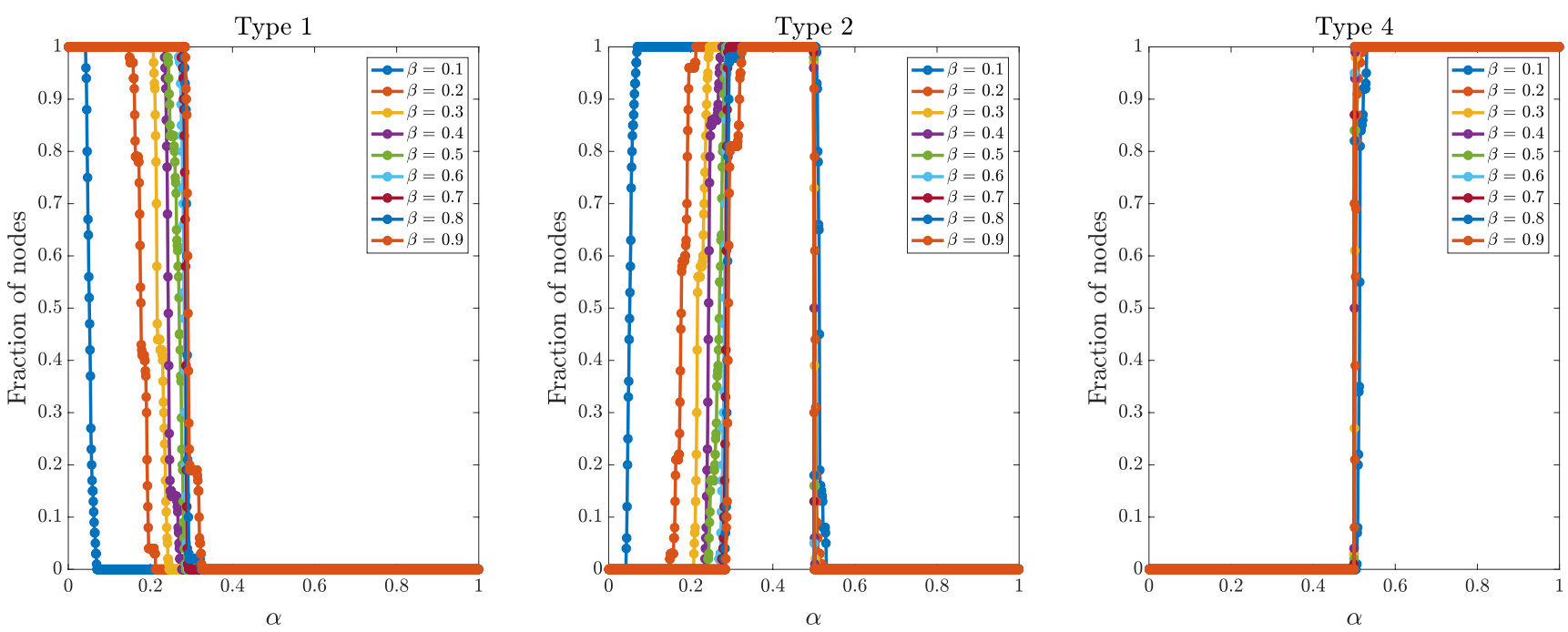

FIG. 7. Fraction of nodes in Watts-Strogatz synthetic networks with $n=100$ nodes (rewiring probability $\beta$ and $500 \beta$ links) such that $\mathcal{P} \mathcal{R}_{\alpha}(i) \subseteq \mathcal{P} \mathcal{R} \mathcal{B}_{\alpha}(i)($ Type 1 , left panel), $x_{\min }<c_{\min } \leq x_{\max } \leq c_{\max }$ (Type 2, central panel), and $\mathcal{P} \mathcal{R} \mathcal{B}_{\alpha}(i) \subset \mathcal{P} \mathcal{R}_{\alpha}(i)$ (Type 4, right panel) for different values of the rewiring probability $\beta$. 

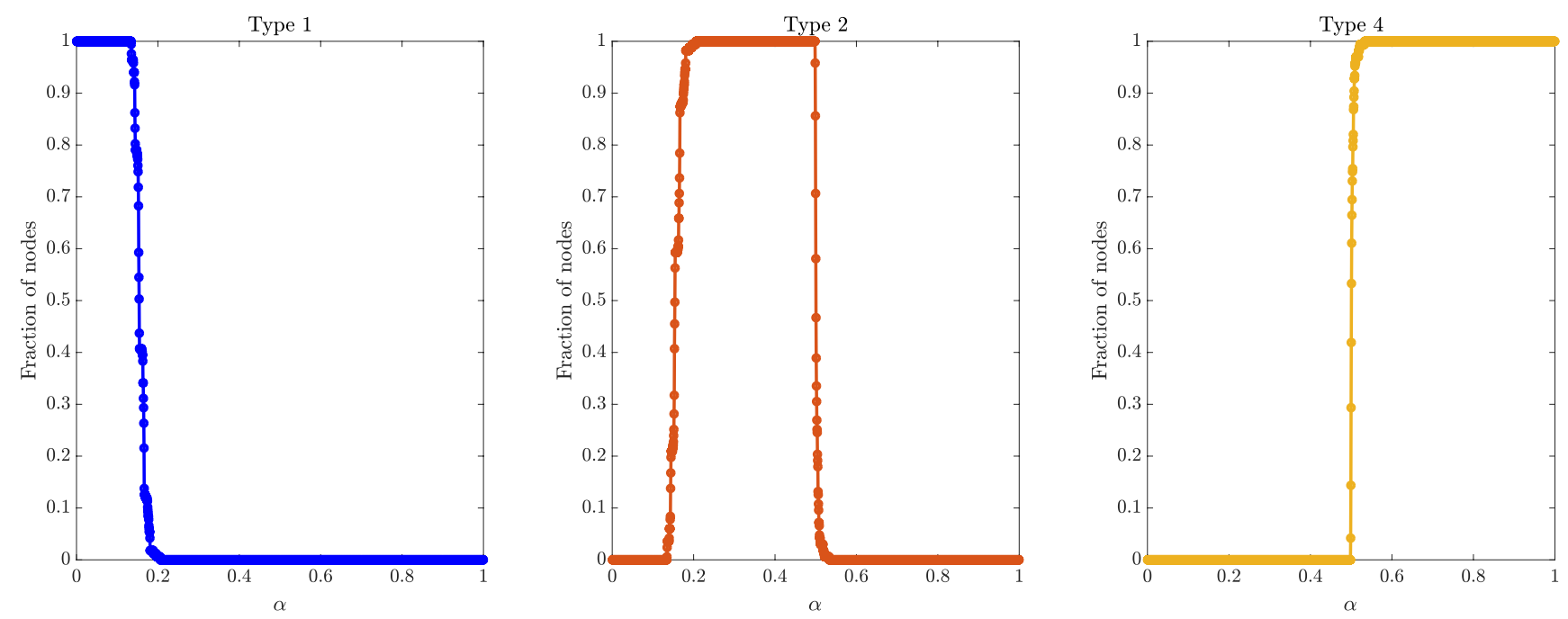

FIG. 8. Fraction of nodes in the giant connected component of Facebook users network of 167 nodes such that $\mathcal{P} \mathcal{R}_{\alpha}(i) \subseteq \mathcal{P} \mathcal{R} \mathcal{B}_{\alpha}(i)\left(\right.$ Type 1 , left panel), $x_{\text {min }}<c_{\text {min }} \leq$ $x_{\max } \leq c_{\max }\left(\right.$ Type 2, central panel), and $\mathcal{P} \mathcal{R} \mathcal{B}_{\alpha}(i) \subset \mathcal{P} \mathcal{R}_{\alpha}(i)$ (Type 4, right panel).

(or friends lists) from Facebook collected from survey participants using an app (see Ref. 63 for more details). If we compute the relative position of intervals $\mathcal{P} \mathcal{R}_{\alpha}(i)$ and $\mathcal{P} \mathcal{R} \mathcal{B}_{\alpha}(i)$ as a function of $\alpha$, Fig. 8 shows that, as it happened for all previous synthetic networks, for small values of $\alpha$ (smaller than 0.2), all nodes have a relative position of intervals $\mathcal{P} \mathcal{R}_{\alpha}(i)$ and $\mathcal{P} \mathcal{R} \mathcal{B}_{\alpha}$ (i) of Type 1, while if $\alpha$ is bigger than a value around 0.5 all nodes have a relative position of intervals of Type 4. If we take values of $\alpha$ in the interplay, then there are again simultaneously nodes with relative positions of intervals of Types 1 and 2, but there are no nodes with relative position of intervals of Type 3.

\section{CONCLUSIONS}

In this paper, we have compared the intervals of variation of two centrality measures known as classic PageRank (PR) and biplex approach PageRank (BPR). The main tool to perform the analysis has been the use of power series and their properties. From the theoretical study (Secs. II and III), we can highlight the following conclusions.

1. The intervals of variation of $P R$ and BPR always have a nonempty intersection. This evidences that the BPR is a reasonable approximation to $\mathrm{PR}$, in the sense that the upper and lower limits can be different, but there is always a zone in where the results are the same.

2. When the damping parameter $\alpha$ is lower than 0.48 , we have shown that the upper limits of the interval for PR are lower than the upper limits of the interval for BPR. This means that when the effects of the teleportation are high (and, therefore, there is a substantial neglect of the topology of the network), the PR is more controllable in its upper zone. In Sec. IV, we have obtained that for small values of $\alpha$, the relative position of the intervals is of Type 1 or 2, that is, consistent with the theoretical analysis.
3. The cases in which the upper limits of the interval for BPR are lower than the upper limits of the interval for PR always happen when $\alpha$ is greater than 0.5 . In particular, for small values of the elements of the row-stochastic matrix $P$ associated with the network, it holds that upper limits of the BPR interval are lower than upper limits of the PR interval when $\alpha$ is greater than 0.5 . The consequences of this fact are remarkable. Note that usually we are interested in the top-K levels of a ranking, that is, the upper limits. Therefore, knowing that the usual value for $\alpha$ is 0.85 , this conclusion means that in this situation, the BPR is more controllable in its higher values than the PR. In Sec. IV, we have obtained that for high values of $\alpha$, the relative position of the intervals is of Type 4 [with no appearance of any interval of Type (3) that is consistent with our theoretical analysis].

4. The theoretical analysis that we have presented in Sec. III is very restrictive for small values of alpha since it requires that alpha must be close to zero. However, for small values of $\alpha$ (and, therefore, high teleportation), in most of our numerical results, it holds that lower limits of the BPR interval are lower than lower limits of the PR interval. This conclusion, together with the previous one, implies that for small values of the damping factor, the interval of variation of the biplex PageRank is contained into the interval of variation of the classic PageRank, that is, $\mathcal{P} \mathcal{R}_{\alpha}(i) \subset \mathcal{P} \mathcal{R} \mathcal{B}_{\alpha}(i)$, that corresponds to the situation that we have denoted as Type 1 . It is interesting to note that this effect was observed in the numerical examples of Ref. 2, where, by the nature of the model, high values of teleportation were preferred.

\section{ACKNOWLEDGMENTS}

This work has been partially supported by the Spanish Ministry of Science, Innovation and Universities under Project 
Nos. PGC2018-101625-B-I00, MTM2016-76808-P, and MTM201784194-P (AEI/FEDER, UE).

\section{REFERENCES}

${ }^{1}$ T. Agryzkov, M. Curado, F. Pedroche, L. Tortosa, and J. F. Vicent, "Extending the adapted PageRank algorithm centrality to multiplex networks with data using the PageRank two-layer approach," Symmetry 11(2), 284 (2019).

${ }^{2}$ T. Agryzkov, F. Pedroche, L. Tortosa, and J. F. Vicent, "Combining the twolayers PageRank approach with the APA centrality in networks with data," ISPRS Int. J. Geo-Inf. 7(12), 480 (2018).

${ }^{3} \mathrm{H}$. Allcott, M. Gentzkow, and C. Yu, "Trends in the diffusion of misinformation on social media," Res. Politics 6(2), 1-8 (2019).

${ }^{4}$ D. Aleja, R. Criado, A. J. García del Amo, Á. Pérez, and M. Romance, "Nonbacktracking PageRank: From the classic model to Hashimoto matrices," Chaos, Solitons Fractals 126, 283 (2019).

${ }^{5}$ F. Arrigo and F. Tudisco, "Multi-dimensional, multilayer, nonlinear and dynamic HITS," in Proceedings of the SIAM International Conference on Data Mining (SIAM, 2019), pp. 369-377.

${ }^{6}$ A. L. Barabási and R. Albert, "Emergence of scaling in random networks," Science 286(5439), 509-512 (1999).

${ }^{7}$ A. Bavelas, “A mathematical model for group structure," Hum. Organ. 7, 16-30 (1948).

${ }^{8}$ A. R. Benson, “Three hypergraph eigenvector centralities," SIAM J. Math. Data Sci. 1(2), 293-312 (2019).

${ }^{9}$ M. Berlingerio, M. Coscia, F. Giannotti, A. Monreale, and D. Pedreschi, "Foundations of multidimensional network analysis," in 2011 International Conference on Advances in Social Networks Analysis and Mining (Kaohsiung, 2011), pp. 485-489.

${ }^{10}$ S. Boccaletti, G. Bianconi, R. Criado, C. I. del Genio, J. Gómez-Gardeñes, M. Romance, I. Sendiña-Nadal, Z. Wang, and M. Zanin, "The structure and dynamics of multilayer networks,” Phys. Rep. 544(1), 1-122 (2014).

${ }^{11}$ P. Boldi and S. Vigna, “Axioms for centrality," Internet Math. 10(3-4), 222-262 (2014).

${ }^{12}$ P. Boldi, M. Santini, and S. Vigna, "PageRank: Functional dependencies," ACM Trans. Inf. Syst. 27(4), 19:1-19:23 (2009).

${ }^{13} \mathrm{P}$. Bonacich, "Factoring and weighting approaches to status scores and clique identification,” J. Math. Sociol. 2(1), 113-120 (1972).

${ }^{14}$ S. P. Borgatti and M. G. Everett, "A graph-theoretic perspective on centrality," Soc. Networks 28(4), 466-484 (2006).

${ }^{15} \mathrm{M}$. Buzzanca, V. Carchiolo, A. Longheu, M. Malgeri, and G. Mangioni, "Black hole metric: Overcoming the PageRank normalization problem," Inf. Sci. 438, 58-72 (2018).

${ }^{16}$ M. De Domenico, A. Solé-Ribalta, E. Omodei, S. Gómez, and A. Arenas, "Ranking in interconnected multilayer networks reveals versatile nodes," Nat. Commun. 6, 6868 (2015).

${ }^{17}$ D. R. DeFord and S. D. Pauls, "A new framework for dynamical models on multiplex networks,” J. Complex Networks 6, 353-381 (2018).

${ }^{18}$ G. M. Del Corso and F. Romani, "A multi-class approach for ranking graph nodes: Models and experiments with incomplete data," Inf. Sci. 329, 619-637 (2016).

${ }^{19}$ N. Dunford and J. T. Schwartz, Linear Operators. Part I (Wiley Classics Library, John Wiley \& Sons, Inc., New York, 1988).

${ }^{20}$ P. Erdös and A. Rényi, “On random graphs I," Publ. Math. Debrecen 6, 290-297 (1959).

${ }^{21}$ E. Estrada and G. Silver, "Accounting for the role of long walks on networks via a new matrix function," J. Math. Anal. Appl. 449(2), 1581-1600 (2017).

${ }^{22}$ L. Festinger, "The analysis of sociograms using matrix algebra," Hum. Relat. 2(2), 153-158 (1949).

${ }^{23} \mathrm{M}$. Fiedler, "A property of eigenvectors of nonnegative symmetric matrices and its application to graph theory," Czech. Math. J. 25(4), 619-633 (1975).

${ }^{24}$ L. R. Ford, Network Flow Theory (RAND Corporation, Santa Monica, CA, 1956).
${ }^{25}$ S. Fortunato, M. Boguñá, A. Flammini, and F. Menczer, "Approximating PageRank from in-degree," in Algorithms and Models for the Web-Graph. WAW 2006, Lecture Notes in Computer Science Vol. 4936, edited by W. Aiello, A. Broder, J. Janssen, and E. Milios (Springer, Berlin, 2006).

${ }^{26}$ L. C. Freeman, "Centrality in social networks: Conceptual clarification," Soc. Networks 1, 215-239 (1979).

${ }^{27}$ L. Ermann, K. M. Frahm, and D. L. Shepelyansky, "Google matrix analysis of directed networks,” Rev. Mod. Phys. 87, 1261 (2015).

${ }^{28}$ K. M. Frahm and D. L. Shepelyanskym, "Ising-PageRank model of opinion formation on social networks," Phys. A Stat. Mech. Appl. 526, 121069 (2019).

${ }^{29}$ E. García, F. Pedroche, and M. Romance, "On the localization of the personalized PageRank of complex networks," Linear Algebra Appl. 439, 640-652 (2013).

${ }^{30}$ D. F. Gleich, P. G. Constantine, A. D. Flaxman, and A. Gunawardana, "Tracking the random surfer: Empirically measured teleportation parameters in pageRank," in Proceedings of the 19th International Conference on World Wide Web Pages (ACM, 2010), pp. 381-390.

${ }^{31}$ C. Gu, X. Jiang, C. Shao, and Z. Chen, "A GMRES-power algorithm for computing PageRank problems,” J. Comput. Appl. Math. 343, 113-123 (2018).

${ }^{32}$ A. Halu, R. J. Mondragón, P. Panzarasa, and G. Bianconi, "Multiplex PageRank," PLoS ONE 8(10), e78293 (2013).

${ }^{33}$ R. A. Horn and C. R. Johnson, Topics in Matrix Analysis (Cambridge University Press, New York, 1991).

${ }^{34}$ J. Iacovacci and G. Bianconi, "Extracting information from multiplex networks," Chaos 26, 065306 (2016).

${ }^{35}$ J. Iacovacci, C. Rahmede, A. Arenas, and G. Bianconi, "Functional multiplex PageRank," Europhys. Lett. 116, 28004 (2016).

${ }^{36} \mathrm{G}$. Iván and V. Grolmusz, "When the web meets the cell: Using personalized PageRank for analyzing protein interaction networks," Bioinformatics 27(3), 405-407 (2011).

${ }^{37}$ K. Kalecky and Y. R. Cho, "PrimAlign: PageRank-inspired Markovian alignment for large biological networks," Bioinformatics 34(13), 537-546 (2018).

${ }^{38}$ L. Katz, Psychometrika 18, 39 (1953).

${ }^{39}$ J. B. Kruskal, "On the shortest spanning subtree of a graph and the traveling salesman problem," in Proceedings of the American Mathematical Society (1956), Vol. 7, No. 1, pp. 48-50.

${ }^{40}$ A. N. Langville and C. D. Meyer, "Deeper inside PageRank," Internet Math. 1(3), 335-380 (2004).

${ }^{41}$ Y. Y. Liu, J. J. Slotine, and A. L. Barabasi, “Controllability of complex networks," Nature 473(7346), 167-173 (2011).

${ }^{42}$ L. Lv, K. Zhang, T. Zhang, D. Bardou, J. Zhang, and Y. Cai, "PageRank centrality for temporal networks,” Phys. Lett. A 383(12), 1215-1222 (2019).

${ }^{43} \mathrm{~J}$. McAuley and J. Leskovec, "Learning to discover social circles in ego networks,” Adv. Neural Inf. Process. Syst. 1, 539-547 (2012).

${ }^{44}$ F. A. Massucci and D. Docampo, "Measuring the academic reputation through citation networks via PageRank," J. Informetr. 13(1), 185-201 (2019).

${ }^{45}$ N. Masuda, M. A. Porter, and R. Lambiotte, "Random walks and diffusion on networks," Phys. Rep. 716-717, 1-58 (2017).

${ }^{46}$ H. Migallón, V. Migallón, and J. Penadés, "Parallel two-stage algorithms for solving the PageRank problem," Adv. Eng. Softw. 125, 188-199 (2018).

${ }^{47}$ M. Newman, Networks: An Introduction (Oxford University Press, 2010).

${ }^{48}$ V. Nicosia, R. Criado, M. Romance, G. Russo, and V. Latora, "Controlling centrality in complex networks," Sci. Rep. 2, 218 (2012).

${ }^{49}$ L. Page, S. Brin, R. Motwani, and T. Winograd, "The PageRank citation ranking: Bridging order to the web,” Tech. Rep. 1999-66, Stanford University, 1998.

${ }^{50}$ F. Pedroche, E. García, M. Romance, and R. Criado, "Sharp estimates for the personalized multiplex PageRank,” J. Comput. Appl. Math. 330, 1030-1040 (2018).

${ }^{51}$ F. Pedroche, L. Tortosa, and J. F. Vicent, "An eigenvector centrality for multiplex networks with data," Symmetry 11(6), 763 (2019).

${ }^{52}$ F. Pedroche, M. Romance, and R. Criado, "A biplex approach to PageRank centrality: From classic to multiplex networks," Chaos 26, 065301 (2016).

${ }^{53}$ C. Sciarra, G. Chiarotti, F. Laio, and L. Ridolfi, "A change of perspective in network centrality,” Sci. Rep. 8, 15269 (2018). 
${ }^{54}$ M. Scholz, J. Pfeiffer, and F. Rothlauf, "Using PageRank for non-personalized default rankings in dynamic markets," Eur. J. Oper. Res. 260(1), 388-401 (2017). ${ }^{55} \mathrm{Y}$. Shen, C. Gu, and P. Zhao, "Structural vulnerability assessment of multienergy system using a PageRank algorithm," Energy Procedia 158, 6466-6471 (2019).

${ }^{56}$ Z. L. Shen, T. Z. Huang, B. Carpentieri, C. Wen, X. M. Gu, and X. Y. Tan, "Offdiagonal low-rank preconditioner for difficult PageRank problems," J. Comput. Appl. Math. 346, 456-470 (2019).

${ }^{57}$ D. L. Shepelyansky and O. V. Zhirovc, "Towards Google matrix of brain," Phys. Lett. A 374, 3206-3209 (2010).

${ }^{58}$ L. Sola, M. Romance, R. Criado, J. Flores, A. Garcia del Amo, and S. Boccaletti, "Eigenvector centrality of nodes in multiplex networks," Chaos 23, 033131 (2013).
${ }^{59}$ Z. Tian, Y. Liu, Y. Zhang, Z.Liu, and M. Tian, "The general inner-outer iteration method based on regular splittings for the PageRank problem," Appl. Math. Comput. 356, 479-501 (2019).

${ }^{60}$ C. Timm and R. Perez, "Social networking infrastructure attacks," in Seven Deadliest Social Network Attacks, edited by C. Timm and R. Perez (Syngress, 2010), pp. 1-22.

${ }^{61}$ D. J. Watts and S. H. Strogatz, "Collective dynamics of small-world networks," Nature 393(6684), 440 (1998).

${ }^{62}$ T. S. Yun, D. Jeong, and S. Park, "Too central to fail systemic risk measure using PageRank algorithm," J. Econ. Behav. Organ. 162, 251-272 (2019).

${ }^{63}$ See file facebook.tar.gz in http://snap.stanford.edu/data/ego-Facebook.html with information about a Dataset of Facebook users' network collected from survey participants using a Facebook app. 